\title{
Sphingosine-1-phosphate receptor-2 deficiency leads to inhibition of macrophage proinflammatory activities and atherosclerosis in apoE-deficient mice
}

\author{
Fei Wang, ${ }^{1}$ Yasuo Okamoto, ${ }^{1}$ Isao Inoki, ${ }^{1,2}$ Kazuaki Yoshioka, ${ }^{1}$ Wa Du, ${ }^{1}$ \\ Xun Qi, ${ }^{1}$ Noriko Takuwa, ${ }^{1,3}$ Koichi Gonda, ${ }^{4}$ Yasuhiko Yamamoto, ${ }^{5}$ \\ Ryunosuke Ohkawa, ${ }^{6}$ Takumi Nishiuchi,7,8 Naotoshi Sugimoto,1 Yutaka Yatomi, 6 \\ Kunitoshi Mitsumori, ${ }^{9}$ Masahide Asano, ${ }^{10}$ Makoto Kinoshita, ${ }^{11}$ and Yoh Takuwa ${ }^{1}$
}

\begin{abstract}
1Department of Physiology and 2Department of Disease Control and Homeostasis, Graduate School of Medicine, Kanazawa University, Kanazawa, Japan.
${ }^{3}$ Department of Health Science and Medicine, Ishikawa Prefectural Nursing University, Kahoku, Japan. ${ }^{4}$ Department of Plastic Surgery,

University of Tokyo Graduate School of Medicine, Tokyo, Japan. 5Department of Vascular Biology, Graduate School of Medicine,

Kanazawa University, Kanazawa, Japan. ${ }^{6}$ Department of Clinical Laboratory Medicine, University of Tokyo Graduate School of Medicine, Tokyo, Japan.

'Division of Life Science, Graduate School of Natural Science and Technology, Kanazawa University, Kanazawa, Japan.

8Division of Functional Genomics, Advanced Science Research Center, Kanazawa University, Kanazawa, Japan.

${ }^{9}$ Department of Veterinary Medicine, Tokyo University of Agricultural Technology, Fuchu, Japan. ${ }^{10 D i v i s i o n ~ o f ~ T r a n s g e n i c ~ A n i m a l ~ S c i e n c e, ~}$

Advanced Science Research Center, Kanazawa University, Kanazawa, Japan. ${ }^{11}$ Department of Medicine, Teikyo University School of Medicine, Tokyo, Japan.
\end{abstract}

\begin{abstract}
Sphingosine-1-phosphate (S1P) is a biologically active sphingolipid that has pleiotropic effects in a variety of cell types including ECs, SMCs, and macrophages, all of which are central to the development of atherosclerosis. It may therefore exert stimulatory and inhibitory effects on atherosclerosis. Here, we investigated the role of the S1P receptor S1PR2 in atherosclerosis by analyzing S1 pr2 $2^{-/-}$mice with an Apoe $\mathrm{A}^{-/-}$background. S1PR2 was expressed in macrophages, ECs, and SMCs in atherosclerotic aortas. In S1 $\mathrm{pr}^{-/-} \mathrm{Apoe}^{-/-}$mice fed a high-cholesterol diet for 4 months, the area of the atherosclerotic plaque was markedly decreased, with reduced macrophage density, increased SMC density, increased eNOS phosphorylation, and downregulation of proinflammatory cytokines compared with $\mathrm{S} 1 \mathrm{pr}^{+/+} \mathrm{Apoe}^{-/-}$mice. Bone marrow chimera experiments indicated a major role for macrophage S1PR2 in atherogenesis. S1 $\mathrm{pr}^{-/-} \mathrm{Apoe}^{-/-}$macrophages showed diminished Rho/Rho kinase/NF-KB (ROCK/NF-KB) activity. Consequently, they also displayed reduced cytokine expression, reduced oxidized LDL uptake, and stimulated cholesterol efflux associated with decreased scavenger receptor expression and increased cholesterol efflux transporter expression. S1 $\mathrm{pr}^{-/-} \mathrm{Apoe}^{-/-}$ECs also showed reduced ROCK and NF- $\kappa B$ activities, with decreased MCP-1 expression and elevated $\mathrm{eNOS}$ phosphorylation.

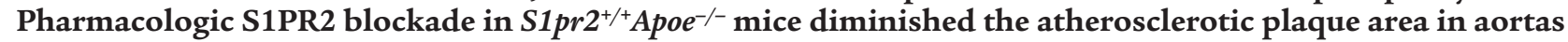
and modified LDL accumulation in macrophages. We conclude therefore that S1PR2 plays a critical role in atherogenesis and may serve as a novel therapeutic target for atherosclerosis.
\end{abstract}

\section{Introduction}

Atherosclerosis is a chronic inflammatory process involving complex interactions of modified lipoproteins, monocyte-derived macrophages or foam cells, T lymphocytes, ECs, and SMCs $(1,2)$. Oxidized LDL (oxLDL) and other triggers induce dysfunction of ECs, which leads to in increased adhesiveness of ECs to leukocytes and production of proinflammatory cytokines including monocyte chemotactic protein-1 (MCP-1), leading to recruitment of monocytes into the intima. These monocytes then differentiate into macrophages, which uptake oxLDL to become foam cells in arterial lesions. The foam cells produce more proinflammatory cytokines with the interactions with ECs and T cells, resulting in further recruitment of monocytes.

Sphingosine-1-phosphate (S1P), a biologically active sphingolipid mediator, exerts pleiotropic effects such as cell proliferation, survival, migration, and cell-cell adhesion in a variety of cell types includ-

Conflict of interest: The authors have declared that no conflict of interest exists. Citation for this article: J Clin Invest. 2010;120(11):3979-3995. doi:10.1172/JCI42315. ing ECs, SMCs, and macrophages (3). S1P is present at the order of $10^{-7}$ to approximately $10^{-6} \mathrm{M}$ concentration in the plasma, largely in forms bound to plasma proteins including HDL and albumin (4), and at lower concentrations in the tissues $(5,6)$. S1P is generated by the phosphorylation of sphingosine by sphingosine kinases 1 (Sphk-1) and 2 (Sphk-2) (7). The major source of plasma S1P is believed to be red blood cells and activated platelets $(7,8)$, which lack the S1Pdegrading enzyme S1P lyase (SPL). In addition, vascular endothelial cells and other types of cells also likely contribute to production of plasma S1P (6). Many of S1P's actions are mediated by 5 members of S1P-specific high-affinity GPCRs (S1PR1-S1PR5) (7). S1P receptor subtypes activate partially overlapping but receptor subtype-specific distinct signaling pathways. Among 3 widely expressed receptor subtypes, S1PR1-3, S1PR1, and S1PR3 couple dominantly to $G_{i}$ to lead to Rac activation and chemotaxis whereas S1PR2 couples mainly to $\mathrm{G}_{12 / 13}$ to result in Rho activation, Rac inhibition, and stimulation of the 3 '-specific phosphoinositide phosphatase, phosphatase, and tensin homolog (PTEN), leading to chemorepulsion (9). We previously demonstrated that S1PR2 in SMCs mediates inhibition of 
PDGF-induced Rac activation and chemotaxis in a Rho-dependent manner (10). Our recent observations also showed that S1PR2 in ECs mediates inhibition of cell migration and angiogenesis, which contrasts with S1PR1's actions in ECs $(11,12)$.

S1PR1 and S1PR2 are the major S1P receptor subtypes that are expressed in monocytes/macrophages (13). Recent studies $(13,14)$ demonstrated that S1P and S1P-containing HDL induce antiinflammatory phenotypes, including inhibition of leukocyte adhesion and proinflammatory cytokine production in monocytes/ macrophages and ECs by stimulating the S1PR1 receptor. In addition, S1P may stimulate or inhibit migration of monocytes/ macrophages via S1PR1 and S1PR2, respectively, depending on relative abundance of these 2 receptors. FTY-720, the phosphorylation product of which is a high-affinity agonist for S1PR1, S1PR3, S1PR4, and S1PR5 but not S1PR2, inhibits development of atherosclerosis in $L d l r$-null mice and Apoe-null mice $(15,16)$. However, it is unknown which S1P receptors exert antiatherogenic effects. There is accumulating evidence that, among the complex array of the GPCR-activated intracellular signaling pathways, Rho and its downstream target the Rho kinase (ROCK) are involved in EC dysfunction, thus contributing to atherogenesis $(17,18)$. It is an intriguing possibility that the $\mathrm{G}_{12 / 13}$ / Rho-coupled receptor S1PR2 could exert distinct effects on atherosclerosis. However, little is thus far known about the role of S1PR2 in atherosclerosis.

We demonstrate here that compound mutant mice lacking S1PR2 and apoE (S1pr2 $2^{--}$Apoe $e^{-/-}$mice) exhibited marked inhibition of atherosclerosis. S1PR2 in monocytes/macrophages plays critical roles in the regulation of oxLDL uptake and cholesterol efflux, monocyte transmigration into the intima, and proinflammatory cytokine production through the mechanisms involving the Rho/ROCK/NF- $\mathrm{BB}$ pathway, contributing to atherogenesis. In addition, S1PR2 in ECs and SMCs appears to participate in atherosclerosis through regulating eNOS activation, proinflammatory cytokine production, and cell migration. We further show that pharmacological blockade of $\mathrm{S} 1 \mathrm{PR} 2$ effectively reduces atherosclerosis. These results indicate that $\mathrm{S} 1 \mathrm{PR} 2$ possesses distinct atherogenic actions, providing what we believe is a novel therapeutic tactic for atherosclerosis.

\section{Results}

Deletion of S1PR2 markedly inhibits the formation of atherosclerotic lesions in Apoe ${ }^{-/}$mice. We generated S1pr2 ${ }^{-/-}$Apoe $e^{-/}$double-knockout mice by intercrossing Apoe $e^{-/}$(C57BL/6 background) and S1pr2-/- mice that had been backcrossed with C57BL/ 6 strain 2 to 6 times to enhance congenicity and to reduce secondary sources of variance. In agreement with a previous report (19), S1pr $2^{-/-}$mice exhibited seizure attacks, which resulted in death around weaning. Repeated backcrossing further increased the death rate. In crossing with Apoe $e^{-/-}$ mice, only $16 \%$ of the weaned N3 offspring were S1pr2-/-Apoe - $^{-1}$ mice. This rate declined to $6 \%$ in the N7 generation (Supplemental Table 1; supplemental material available online with this article; doi:10.1172/JCI42315DS1). Therefore, it was difficult to obtain a large enough number of $S 1$ pr2 $2^{-1-} A p_{0 e^{-/-}}$mice that had been further backcrossed with C57BL/6 mice $(n>7)$.

S1pr2 ${ }^{-/-} \mathrm{Apoe}^{-/-}$mice and their S1pr2 ${ }^{+/+} \mathrm{Apoe}^{-/-}$littermates were fed a high-cholesterol diet (HCD) for 8 weeks or 16 weeks. The food intake, body weight gain, plasma lipid values, and plasma lipoprotein profiles were similar between S1pr2 ${ }^{+/+}$Apoe $^{-/-}$and S1pr2-/-Apoe ${ }^{-/-}$ mice (Supplemental Tables 2 and 3 and Supplemental Figure 1A). Serum alanine aminotransferase (ALT), a marker of liver injury, in both S1pr2 $2^{-/-} \mathrm{Apoe}^{-/-}$and S1 pr2 $2^{+/+} \mathrm{Apoe}^{-/-}$mice fed HCD was similar to that in $\mathrm{S} 1 \mathrm{pr} \mathrm{2}^{+/+} \mathrm{Apoe}^{-/-}$mice fed regular chow, and liver histology in HCD-fed mice did not show signs of liver damage as evaluated with H\&E staining (Supplemental Figure 1, B and C). There was no difference in plasma S1P concentrations between S1pr2-/-Apoe $\mathrm{e}^{-/-}$ and S1pr2 ${ }^{+/+}$Apoe $^{-/-}$mice (Supplemental Table 2).

Atherosclerotic lesions in the aorta were analyzed by en face analysis of the spread total aorta and analysis of cross sections of the aorta. The en face plaque area of the total aorta was reduced by $45 \%-55 \%$ in both male and female S1pr2 $2^{-/-}$Apoe $e^{--}$mice (N3 generation) after 16 weeks but not 8 weeks of HCD compared with S1pr2 ${ }^{+/+} A p o e^{-/-}$ mice (Figure 1A and Supplemental Figure 2A); in S1pr2 ${ }^{-/-} \mathrm{Apoe}^{-/-}$ mice, the progression of the plaque formation after 8 weeks of HCD was nearly abolished. The cross-sectional plaque area of the abdominal aorta was also reduced in S1pr2-/-Apoe - $^{-/}$mice compared with S1pr2 ${ }^{+/+} A p^{-e^{-/}}$mice (Figure 1C). Male S1pr2 ${ }^{-/-}$Apoe $e^{-/-}$ mice of the N7 generation exhibited more than $70 \%$ reductions in the plaque area compared with $S 1 p r 2^{+/+} A p o e^{-/-}$mice (Figure 1B). The plaque area in $S 1 \mathrm{pr}^{+/-} \mathrm{Apoe^{-/- }}$ mice was intermediate between S1pr2 ${ }^{-/-} \mathrm{Apoe}^{-/-}$and S1pr2 ${ }^{+/+} \mathrm{Apoe}^{-/-}$mice, indicating that S1PR2 has a gene dose-dependent proatherogenic effect. Collagen deposition in the plaque area was similar between $S 1 \mathrm{pr}^{-{ }^{--}} \mathrm{Apoe^{-- }}$ and $S 1 \mathrm{pr}^{+/+} A \mathrm{poe}^{-/-}$mice (N3 generation) (Figure 1D), but the infiltration of Mac-3-positive macrophages into the plaque areas was relatively reduced in S1pr2-/-Apoe $\mathrm{e}^{-/-}$mice compared with $\mathrm{S} 1 \mathrm{pr} \mathrm{P}^{+/+} \mathrm{Apoe^{-/- }}$ mice (Figure 1E), whereas $\alpha$-SMA-positive SMCs in the intima were relatively abundant in $S 1 p r 2^{-/-} A_{p o e^{-/-}}$mice compared with $S 1 \mathrm{pr}^{+/+}$Apoe ${ }^{-/-}$mice (Figure 1F). No difference in the abundance of CD3-positive T cells was observed between S1pr2 ${ }^{-/-} \mathrm{Apoe}^{-/-}$and $S 1 \mathrm{pr}^{+/+}$Apoe $^{-/-}$mice (Figure $1 \mathrm{G}$ ). The density of activated mast cells, which were found largely in the adventitia, was reduced in S1pr2 $2^{-/-}$Apoe $e^{-/-}$mice compared with S1pr2 ${ }^{+/+}$Apoe $^{-/-}$mice (Figure $1 \mathrm{H}$ ). These histological features suggest the increased stability of plaques in S1pr2 ${ }^{-/-}$Apoe ${ }^{-/-}$mice compared with S1pr2 ${ }^{+/+} \mathrm{Apoe}^{-/-}$mice.

S1PR2 deficiency caused downregulation of proinflammatory cytokines and VCAM-1, and stimulation of eNOS phosphorylation in the aorta. We analyzed mRNA expression of proinflammatory and antiinflammatory cytokines and adhesion molecules in the aortas from S1pr2 $2^{-/-} \mathrm{Apoe}^{-/-}$and S1pr2 ${ }^{+/+}$Apoe $e^{-/-}$mice. Real-time PCR analysis showed that mRNA expression levels of TNF- $\alpha$, IL-6, IFN- $\gamma$, and MCP-1 corrected for $18 \mathrm{~S}$ ribosomal RNA levels were all diminished

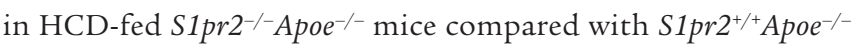
mice whereas that of antiinflammatory IL-10 was higher in S1pr2 $2^{-/}$Apoe $e^{-/}$mice (Figure 2A). The cytokine mRNA expression that was corrected for CD3 mRNA, which likely reflects plaque burdens (Supplemental Figure 2C), also showed differences similar to those corrected for $18 \mathrm{~S}$ ribosomal RNA levels (Figure 2A) between S1pr2 ${ }^{-/-}$Apoe $e^{-/-}$and $S 1 p r 2^{+/+} A p o e^{-/-}$mice, suggesting that the cytokine expression changes reflected an S1PR2 function rather than differences in plaque mass. The mRNA expression of VCAM-1 but not ICAM-1 was reduced in the aorta of S1 pr2-/-Apoe - $^{--}$ mice compared with $S 1 p r 2^{+/+} A p o e^{-/-}$mice (Figure 2B). Immunohistochemistry showed that the expression of VCAM-1 in the neointima was decreased in S1pr2 ${ }^{-/}$Apoe ${ }^{-/}$mice compared with $\mathrm{S} 1 \mathrm{pr}^{+/+} \mathrm{Apoe}^{-/-}$mice but that in the endothelium it was similar in both kinds of mice (Figure 2C). The expression of ICAM-1 in the endothelium and the intima was not different between S1pr2 $2^{-/-}$Apoe $e^{-/-}$and S1pr2 ${ }^{+/} A$ poe $^{-/-}$mice (Figure 2C). Double immunofluorescence by using anti-VCAM-1 and either antiCD31, anti-CCR2, or anti-Mac3 showed that endothelial cells and CCR2- and Mac3-positive intimal macrophages express 
A

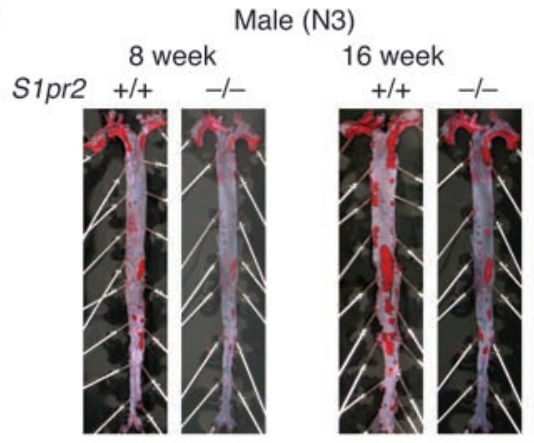

B

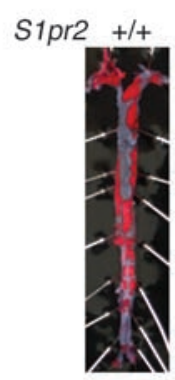

Male (N7)

16 week

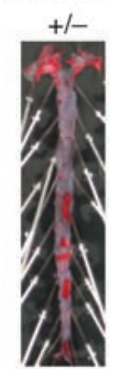

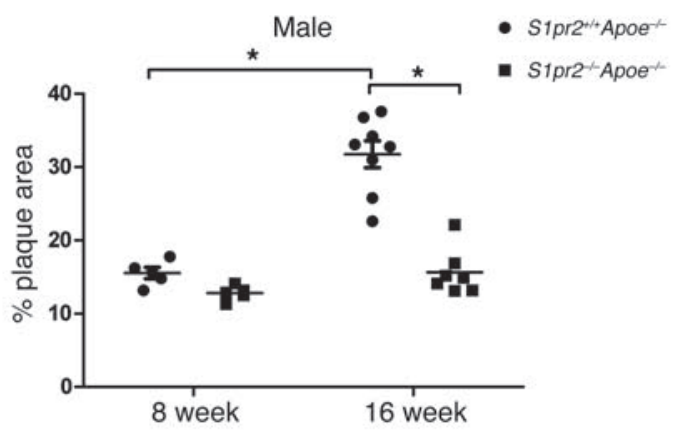
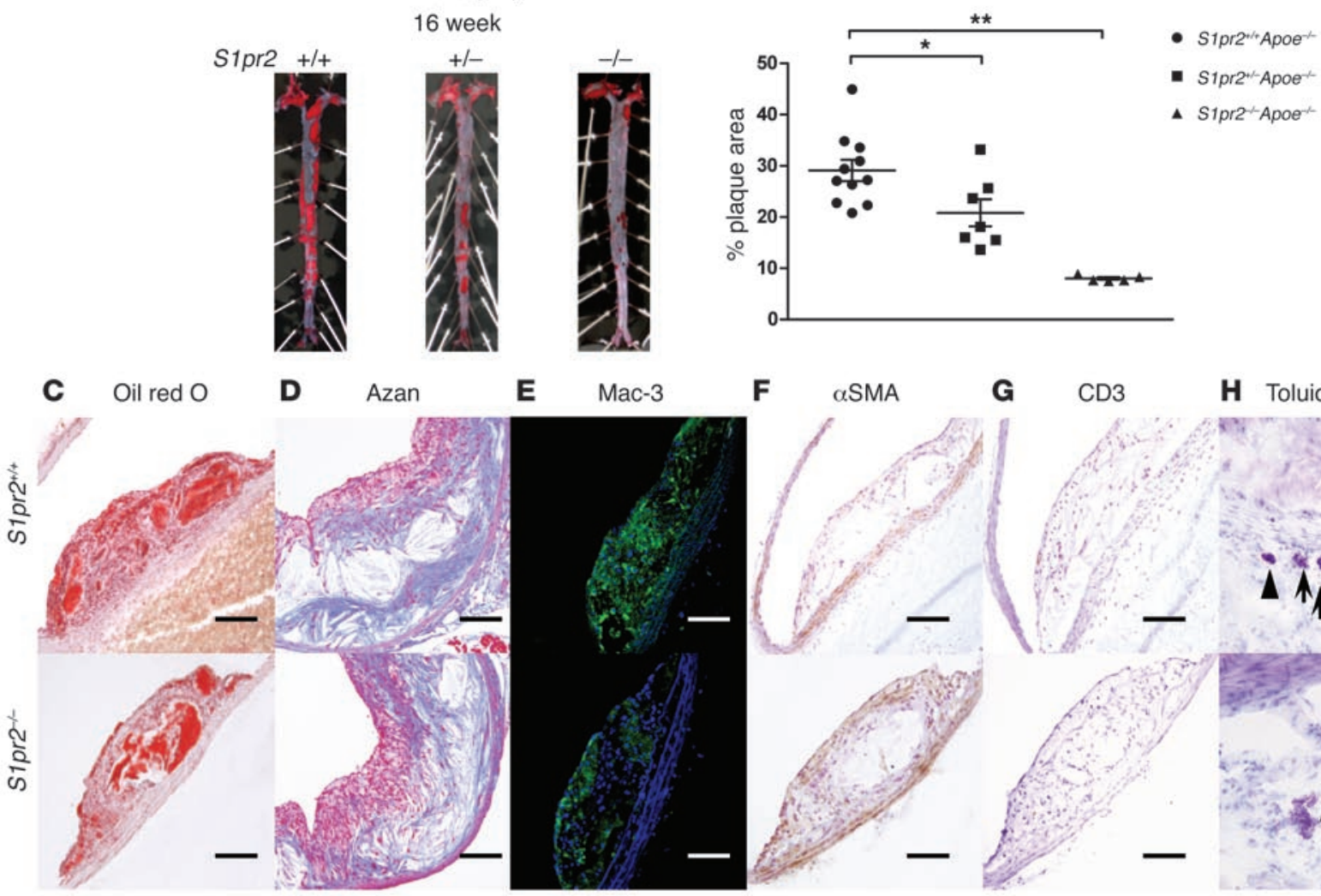

E

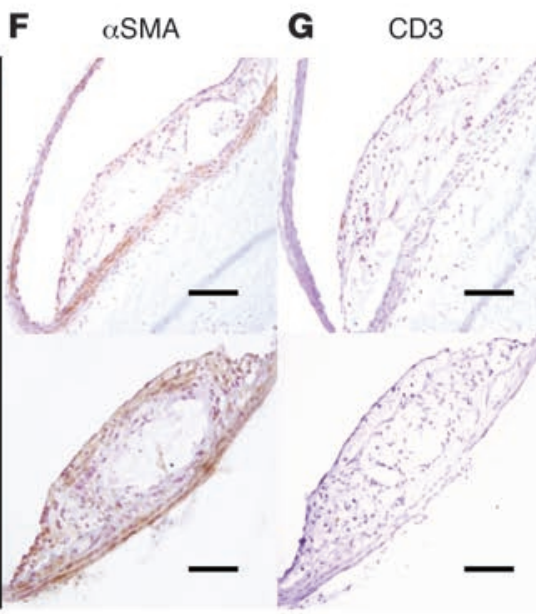

H Toluidine blue
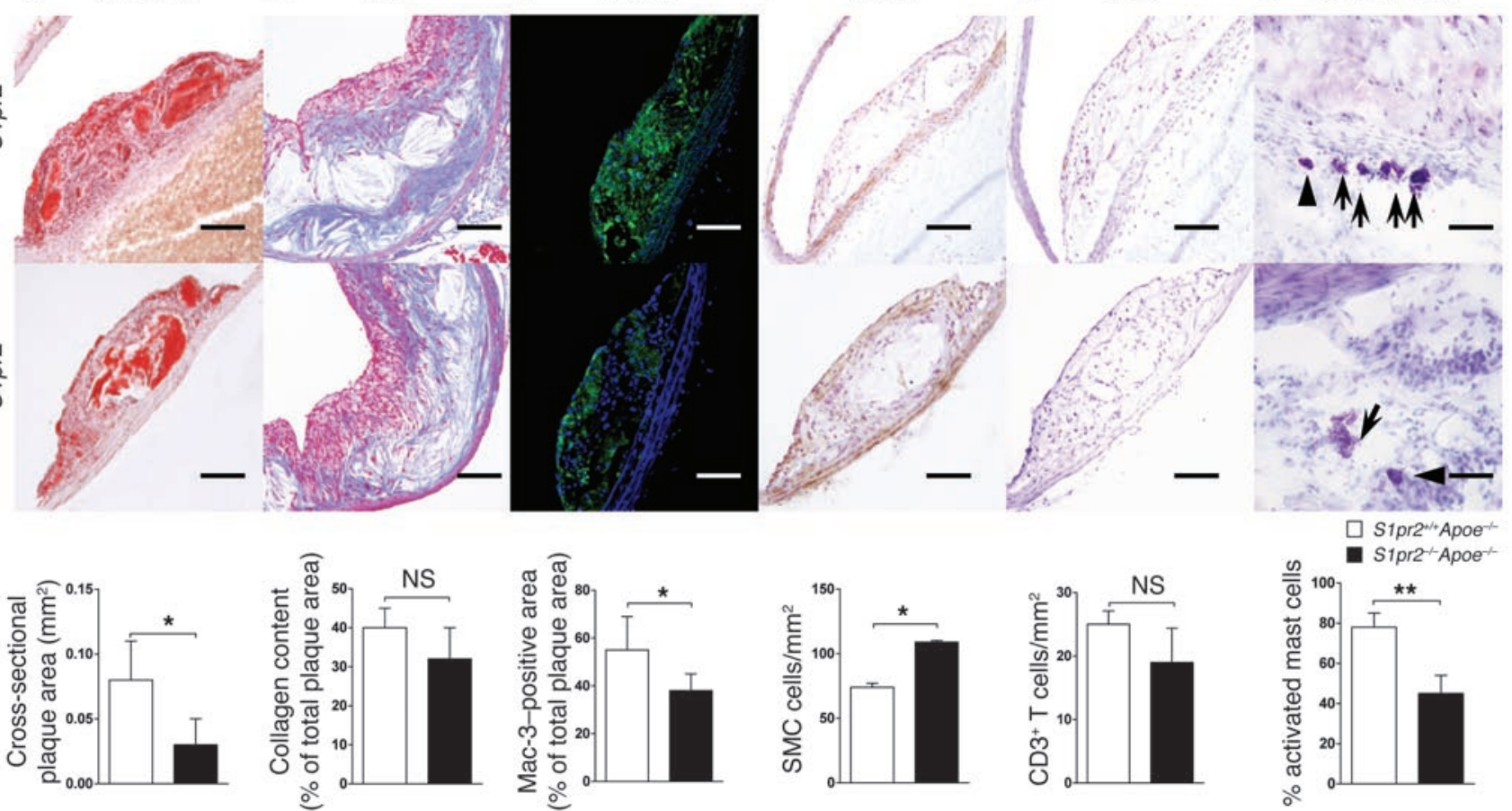

Figure 1

Decreased atherogenesis in mice lacking S1PR2. (A and B) Representative oil red O staining of spread aortas from $S 1 p r 2^{+/+} A p o e^{-/-}(n=13)$ and S1pr2 ${ }^{-/}$Apoe $^{-/-}(n=12)$ mice (N3 generation) after 8 and 16 weeks of HCD $(\mathbf{A})$ and S1pr2 $2^{+/+} A p o e^{-/}(n=11), S 1 p r 2^{+/-} A p o e^{-/-}(n=7)$, and S1pr2 ${ }^{-l-}$ Apoe ${ }^{-/-}$mice $(n=5)$ (N7 generation) after 16 weeks of HCD (B). Quantification of plaque areas is shown (right). ${ }^{\star} P<0.05 ;{ }^{\star \star} P<0.01$. (C-H) Histological analysis of the sections of the aortas from S1 pr2 ${ }^{+/+} A p o e^{-/-}$and S1pr2 $2^{--}$Apoe ${ }^{-/-}$mice (N3 generation). Oil red O staining (C); Azan staining (D); immunostaining of Mac-3 (E), $\alpha-S M A(F)$, and CD3 (G); and toluidine blue staining (arrowhead and arrows denote resting and activated mast cells, respectively) $(\mathbf{H})$. Representative views are shown in the upper panels. Scale bars: $50 \mu \mathrm{m}$. Quantified data are shown in the lower panels $\left(n=5\right.$ each). ${ }^{\star} P<0.05 ;{ }^{*} P<0.01$. Data are expressed as mean \pm SEM. 

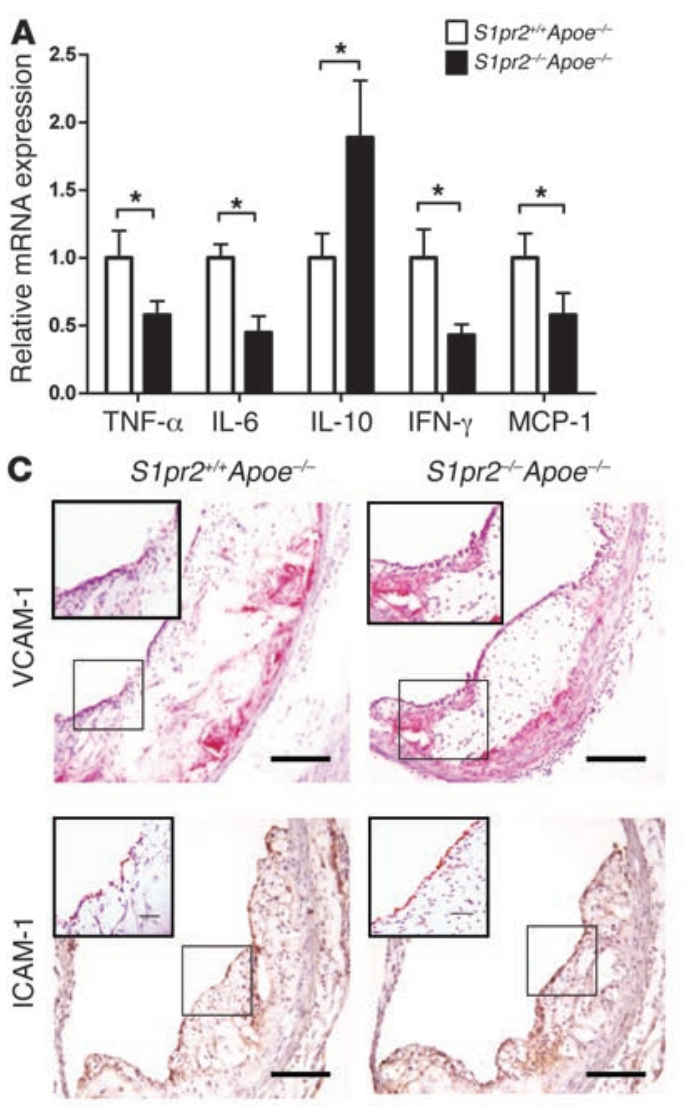

B
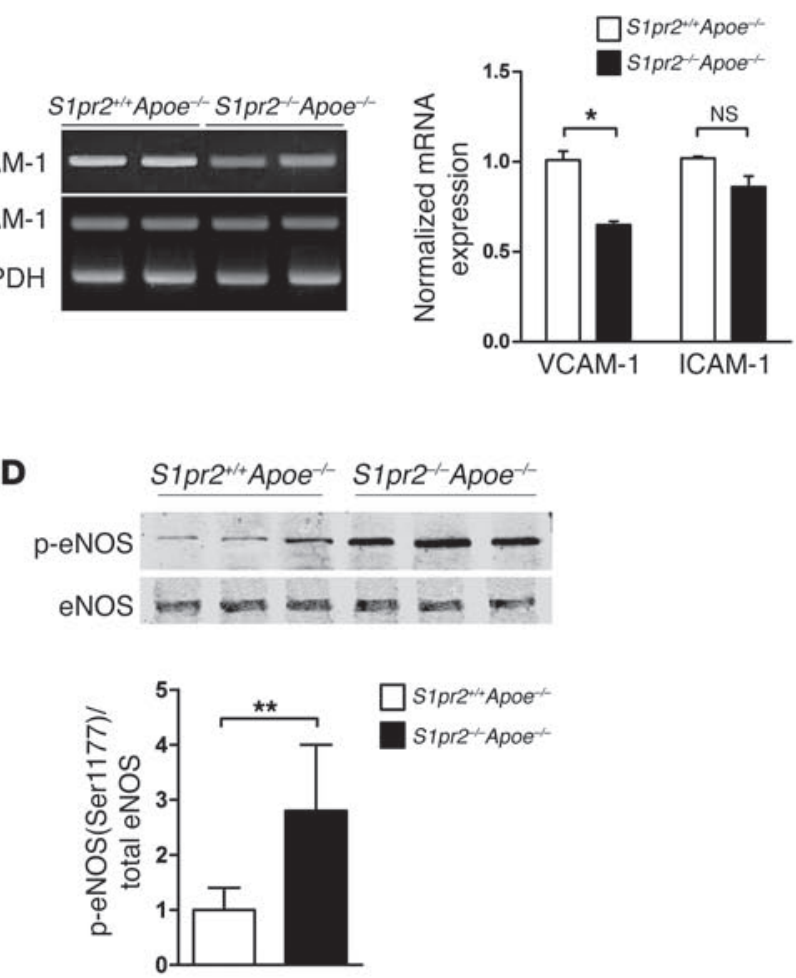

Figure 2

S1PR2 deficiency inhibits the expression of inflammatory cytokines and adhesion molecules and stimulates the expression of an antiinflammatory cytokine and phosphorylation of eNOS in the aorta of $A p o e^{-/-}$mice. (A) mRNA expression levels of TNF- $\alpha$, IL-6, IL-10, IFN- $\gamma$, and MCP-1 $\left(n=5\right.$ each). (B) mRNA expression levels of VCAM-1 and ICAM-1 $\left(n=4\right.$ each). mRNA expression levels in the aortas from S1pr2 ${ }^{+/+} A p o e^{-/-}$and S1 pr2 ${ }^{--}$Apoe $^{-l-}$ mice fed HCD for 12 weeks were determined by real-time PCR in A and semiquantitative RT-PCR in B. 18S rRNA (for real-time $\mathrm{PCR}$ ) and GAPDH (for RT-PCR) were used as internal controls. Data are expressed as the ratio of the values in S1pr2 ${ }^{-/-}$Apoe ${ }^{-/-}$mice over S1pr2 ${ }^{+/} \mathrm{Apoe}^{-/-}$mice. ${ }^{*} P<0.05$. (C) Representative immunostaining for VCAM-1 (upper panels) and ICAM-1 (lower panels) in the aortic sinus

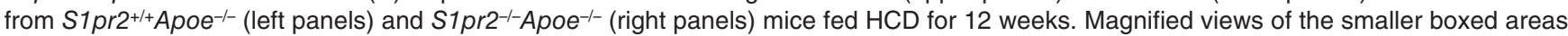
are shown in the insets (left upper of each panel). Scale bars: $100 \mu \mathrm{m}$. (D) Phosphorylation of eNOS in the aortas from S1pr2 ${ }^{+/+} A p o e^{-/-}$and S1 pr2 ${ }^{-/}$Apoe $^{-/}$mice fed HCD for 12 weeks ( $n=3$ each). The extracts of the tissues were subjected to Western blotting, and quantified data are shown in the right panel. ${ }^{* *} P<0.01$. Data are expressed as mean \pm SEM.

VCAM-1 in atherosclerotic lesions (Supplemental Figure 3, A-C). It is reported that during the early stages of atheroma formation, there is a dramatic decrease in endothelium-derived $\mathrm{NO}$, which is a potent vasodilator and antiatherogenic mediator (20). The protein expression of eNOS in the aorta was similar between S1pr2 $2^{-/-} \mathrm{Apoe}^{-/-}$and S1pr2 ${ }^{+/+} \mathrm{Apoe}^{-/-}$mice (Figure 2D). However, the extent of phosphorylation of eNOS at Ser ${ }^{1177}$, which is the activating phosphorylation site, was nearly 3 -fold increased in S1pr2 $2^{-/-}$Apoe $e^{-/-}$mice compared with S1pr2 ${ }^{+/+}$Apoe $^{-/-}$mice, suggesting that stimulation of eNOS is involved in downregulation of the proinflammatory cytokines and VCAM-1 and inhibition of plaque formation.

S1PR2 is expressed in macrophages, SMCs, and ECs in atherosclerotic lesions. We studied the expression of S1PR2 in atherosclerotic lesions by using 5-bromo-4-chloro-3-indolyl- $\beta$-D-galactopyranoside (X-gal) staining of the aortic tissues from S1pr2 ${ }^{\mathrm{LacZ} / \mathrm{LacZ}} \mathrm{Apoe}^{-/-}$mice, where $\beta$-gal (LacZ) is knocked in at the locus of S1pr2 allele and $\beta$-gal gene expression is driven by endogenous S1PR2 promoter. We recently showed that S1PR2 is expressed mainly in ECs and SMCs of blood vessels in a variety of normal organs (12). Consistent with this, ECs and SMCs in the nonatherosclerotic aorta of S1pr2 ${ }^{\mathrm{LacZ} / \mathrm{LacZ}} \mathrm{Apoe} e^{+/+}$ mice were positive for X-gal staining (Supplemental Figure $4 A$ ). In the aortic sinus of S1pr2 ${ }^{\mathrm{LaCZ} / \mathrm{LaCZ}} \mathrm{Apoe}^{-/-}$mice fed HCD, the plaque area was strongly positive for X-gal staining (Supplemental Figure 4B); double staining with either anti-Mac-3, anti- $\alpha$-SMA, or anti-CD31 immunohistochemistry and X-gal staining showed that macrophages and SMCs in the plaque lesions and ECs expressed S1PR2 (Supplemental Figure 4, C-E). Real-time PCR analysis showed that the mRNA expression of the 2 other S1P receptors, S1PR1 and S1PR3, and the S1P synthesizing and degradation enzymes including Sphk-1, Sphk-2, SPL, and S1P phosphatase 1 (SPP1) in the aorta were not different between S1pr2 ${ }^{-/-} \mathrm{Apoe}^{-/-}$and S1pr2 ${ }^{+/+} \mathrm{Apoe}^{-/-}$mice (Supplemental Figure 4F).

Deletion of S1PR2 in BM cells inbibits the atherosclerotic lesion formation. We studied the role of S1PR2 in BM-derived cells for atherosclerosis by using a BM-transplantation technique. $S 1 \mathrm{pr}^{+/+} \mathrm{Apoe}^{-/-}$mice that had undergone transplantation of BM from S1pr2-/-Apoe $\mathrm{e}^{-/-}$mice showed reduced en face plaque areas of 
A

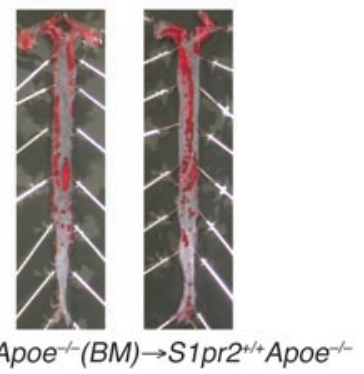

$\mathrm{S}_{\mathrm{pr}} \mathrm{2}^{+/+} \mathrm{Apoe}^{-/-}(\mathrm{BM}) \rightarrow \mathrm{S}_{\mathrm{pr}} \mathrm{2}^{+/+} \mathrm{Apoe} \mathrm{e}^{-/-}$

B $S 1 p r 2^{+/ 4} \mathrm{Apoe}^{-/-} \rightarrow \mathrm{S}_{1 p r 2^{+/}} \mathrm{Apoe}^{-/-}$

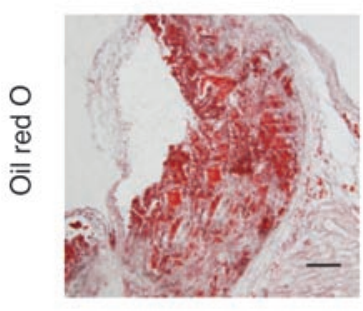

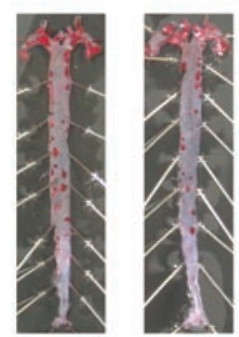

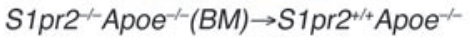
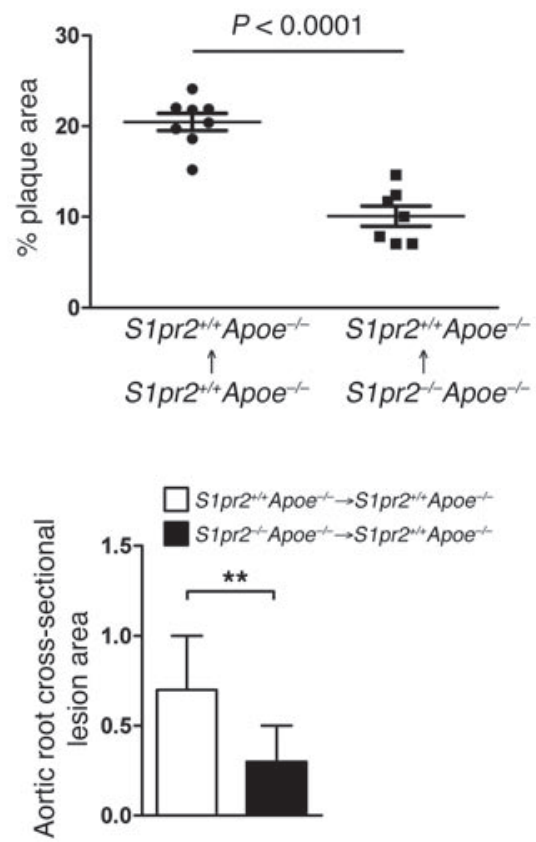

\section{Figure 3}

Deletion of S1PR2 in BM cells inhibits the formation of atherosclerotic lesions. (A) Representative oil red O staining of spread aortas from S1pr2 ${ }^{+/+} A p o e^{-/-}$mice that received transplantation of BM from S1pr2 ${ }^{+/+} A p o e^{-/-}$(left panels) $(n=8)$ and S1pr2 $2^{-/-}$Apoe ${ }^{-/-}($right panels) $(n=7)$ donors 5 weeks prior to 12 weeks of HCD. Quantified plaque areas are shown (right). (B) Oil red O staining views of aortic cross sections of S1pr2 ${ }^{+/+} \mathrm{Apoe}^{-/-}$mice reconstituted with S1pr2 ${ }^{+/+} \mathrm{Apoe} \mathrm{e}^{-/} \mathrm{BM}$ or S1pr2 ${ }^{-/} \mathrm{Apoe^{-/ }}$ BM. Quantified stained areas are shown (right). ${ }^{* \star} P<0.01 . \mathrm{Scale}$ bars: $50 \mu \mathrm{m}$. Data are expressed as mean \pm SEM.

the total aorta after 12 weeks of HCD compared with mice that had received S1pr2 ${ }^{+/+} \mathrm{Apoe}^{-/-} \mathrm{BM}$ (Figure 3A). The cross-sectional plaque area in the abdominal aorta was also reduced in mice that had been transplanted with S1pr2-/-Apoe-/- BM compared with mice that received S1pr2 ${ }^{+/+}$Apoe $^{-/-}$BM (Figure 3B).

S1pr2-deficient macrophages show decreased oxLDL uptake and foam cell formation. We studied in vitro oxLDL uptake in BM-derived macrophages from S1pr2 $2^{-/-} A p o e^{-/-}$and $S 1 p r 2^{+/+} A p o e^{-/-}$mice. BMderived macrophages from $S 1 \mathrm{pr}^{+/+} A \mathrm{poe}^{-/-}$mice expressed S1PR1 and S1PR2 but not other S1P receptor subtypes (Supplemental Figure 5A). BM-derived macrophages from S1pr2 $2^{-/-} A p o e^{-/-}$mice expressed a level of S1PR1 mRNA similar to those of S1 pr2 ${ }^{+/+} \mathrm{Apoe}^{-/-}$ macrophages but lacked S1PR2 mRNA expression. After 6 hours of incubation of macrophages in oxLDL-containing medium, there were approximately half as many oil red O-positive S1 pr2-/-Apoe-/macrophages as $S 1 p r 2^{+/+} A p o e^{-/-}$macrophages (Figure 4, A and B). We also analyzed modified LDL uptake by using DiI-labeled acetylated LDL (DiI-acLDL). Accumulation of DiI-labeled acLDL in S1pr2 $2^{-/}$Apoe ${ }^{-/-}$macrophages was about half of that in S1pr2 ${ }^{+/+}$Apoe $e^{-/}$ macrophages (Figure 4, C and D), similar to the results in oxLDL uptake (Figure 4, A and B). Cholesterol efflux in the presence of HDL from ${ }^{3} \mathrm{H}$-cholesterol-loaded S1pr2 $2^{-/-}$Apoe $^{-/-}$macrophages was increased compared with $S 1 \mathrm{pr}^{+/+}$Apoe ${ }^{-/-}$macrophages (Figure 4E). These observations show that S1PR2 deficiency in macrophages leads to a reduction of modified LDL uptake and stimulation of cholesterol efflux, and thus inhibition of foam cell formation. We also studied phagocytotic capacity by examining the phagocytosis of fluorescent polystyrene microspheres. Phagocytotic activity was reduced in S1pr2 $2^{-/-} \mathrm{Apoe}^{-/-}$macrophages compared with S1pr2 ${ }^{+/+}$Apoe $^{-/-}$macrophages (Supplemental Figure 6).
We investigated the potential mechanisms underlying decreased modified LDL uptake and stimulated cholesterol efflux in S1pr2deficient macrophages. The mRNA and protein expression of CD36 and scavenger receptor class A (SR-A), which mediate uptake of modified lipoproteins in macrophages $(21,22)$, was decreased in BMderived S1pr $2^{-/-}$ppoe $^{-/-}$macrophages compared with S1pr $2^{+/+}$Apoe $^{-/-}$ macrophages (Figure 5, A and C), which was consistent with reduced oxLDL uptake in S1pr2 ${ }^{-/-}$Apoe ${ }^{-/-}$macrophages (Figure 4, A and B). However, the mRNA expression of scavenger receptor class B1 (SR-B1) was rather increased in S1pr2 $2^{-/-}$Apoe $e^{-/-}$macrophages compared with $S 1 \mathrm{pr}^{+/+} \mathrm{Apoe}^{-/-}$macrophages. The mRNA and protein expression of the cholesterol transporters ABCA1 and ABCG1, which mediate the cholesterol efflux from macrophages $(23,24)$, was elevated in S1pr2-/-Apoe $e^{-/-}$macrophages compared with S1pr2 ${ }^{+/+} \mathrm{Apoe}^{-/-}$macrophages (Figure 5, B and C). Moreover, we observed that the mRNA expression of the transcription factors, liver X receptor $\alpha(\operatorname{LXR} \alpha)$ and LXR $\beta$ but not PPAR $\gamma$, which are implicated in upregulation of $A B C A 1$ and $A B C G 1$ gene expression $(25,26)$, was increased (Figure 5D). These observations collectively suggest that S1PR2 deficiency in macrophages leads to downregulation of the lipoprotein scavenger receptors SR-A and CD36 and upregulation of the cholesterol transporters $\mathrm{ABCA} 1$ and $\mathrm{ABCG} 1$, the latter of which likely involves the upregulation of $\operatorname{LXR} \alpha$ and $\operatorname{LXR} \beta$.

Because larger amounts of macrophages were required for the following experiments, we employed peritoneal macrophages, in which S1PR2 deficiency recapitulated the reduced oxLDL uptake and the scavenger receptor (CD36) mRNA expression as well as the increased mRNA expression of the ABC transporters (ABCA1 and ABCG1) and the upstream transcriptional regulator (LXR $\alpha$ ) (data not shown). Peritoneal macrophages from S1pr $2^{+/+} A p o e^{-/-}$mice 
A

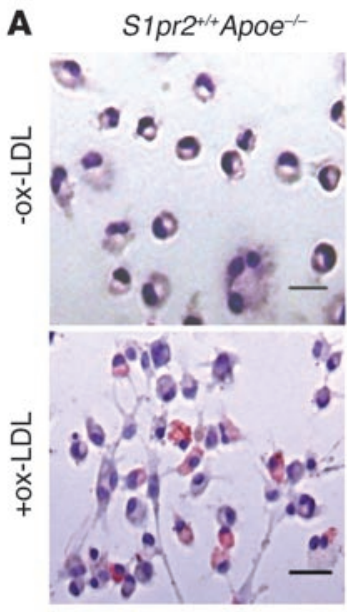

C $\quad$ S1pr2 ${ }^{+/+} \mathrm{Apoe}^{-\alpha}$

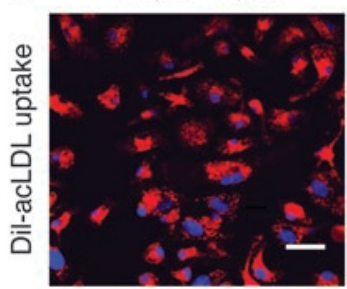

E

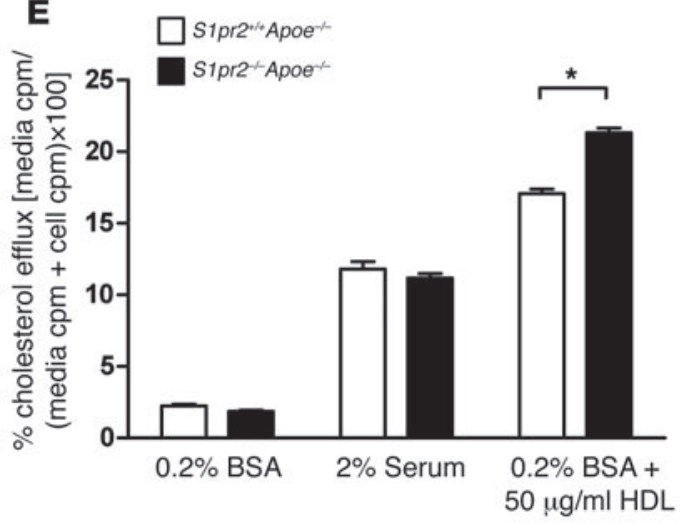

B
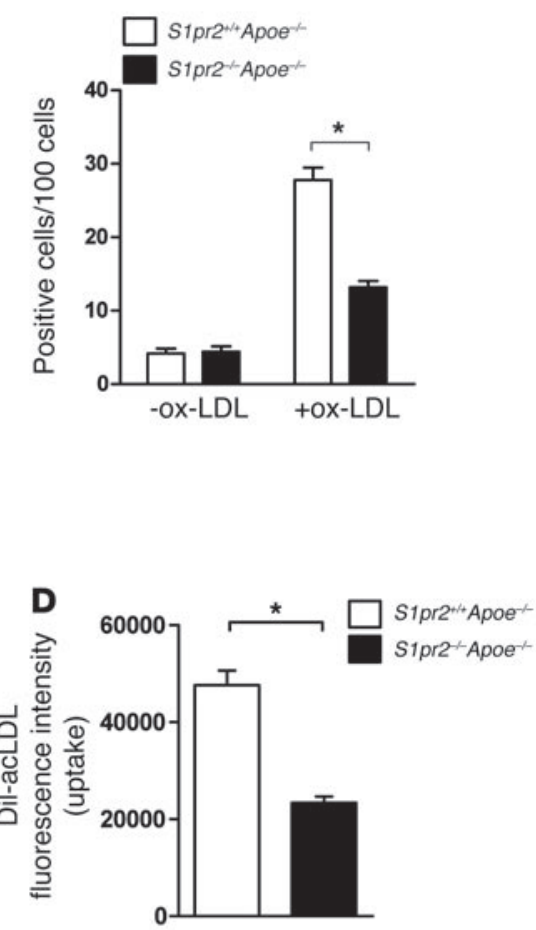

Figure 4

S1PR2 deficiency inhibits uptake of modified LDL and stimulates cholesterol efflux in BM-derived macrophages. (A) Foam cell formation assay. Macrophages from S1pr2 ${ }^{+/+} \mathrm{Apoe}^{-/-}$(left panels) and S1 pr2 ${ }^{-/-} \mathrm{Apoe}^{-/-}$(right panels) mice were exposed to oxLDL for 6 hours. Cells containing oil red $\mathrm{O}-$ positive fat droplets were considered foam cells. Scale bars: $20 \mu \mathrm{m}$. (B) Quantified data of oil red O-positive cells. ${ }^{*} P<0.05$. (C) Uptake of Dil-labeled acetylated-LDL (ac-LDL). Macrophages from S1pr2 ${ }^{+/+} \mathrm{Apoe}^{-/-}$(left panel) and S1 pr2 ${ }^{-/-}$Apoe $^{-/-}$(right panel) mice were incubated with Dil-labeled ac-LDL for 4 hours. Scale bars: $20 \mu \mathrm{m}$. (D) Quantified data of Dil-labeled ac-LDL uptake is shown. ( $n=3$ each). ${ }^{*} P<0.05$. (E) Cholesterol efflux from cholesterol-loaded macrophages. Macrophages from S1pr2 ${ }^{+/+} A p o e^{-/-}$(white bars) and S1 pr2--Apoe-/- (black bars) mice were loaded with $\left[{ }^{3} \mathrm{H}\right]$-labeled cholesterol. Cholesterol-loaded macrophages were incubated in the media containing either $0.2 \%$ BSA, $2 \%$ serum (FBS), or $50 \mu \mathrm{g} / \mathrm{ml} \mathrm{HDL}$ as acceptor for 6 hours. The media and cells were collected for counting $\left[{ }^{3} \mathrm{H}\right]$ radioactivity ( $n=3$ each). ${ }^{*} P<0.05$. Data are expressed as mean \pm SEM. expressed mRNAs of all S1PR1-S1PR5 (Supplemental Figure 5A). S1pr2 $2^{-/} \mathrm{Apoe}^{-/-}$macrophages expressed levels of these receptors similar to those of S1 $\mathrm{pr}^{+/+}$Apoe $e^{-/-}$macrophages, but lacked S1PR2. Consistent with the similar levels of S1PR1 expression in both macrophage types, phosphorylated FTY-720 stimulated Rac comparably in S1pr2 $2^{+/+} \mathrm{Apoe}^{-/-}$and S1pr2 ${ }^{-/-} \mathrm{Apoe}^{-/-}$macrophages (Supplemental Figure 5B). It has been reported that defective uptake of oxLDL and enhanced cholesterol efflux delayed the formation of foam cells and improved survival of macrophages from toxicity of overloaded cholesterol (27). The unstimulated, basal level of Akt phosphorylation, a cell survival signal, was similar between serumdeprived S1pr2 $2^{+/} A p_{0 e^{-/-}}$and S1pr2 $2^{--}$Apoe $e^{-/}$peritoneal macrophages (Supplemental Figure 7A). The addition of S1P decreased Akt phosphorylation in S1pr2 ${ }^{+/+} A \mathrm{poe}^{-/-}$macrophages, whereas S1P increased Akt phosphorylation in S1pr2 $2^{--}$Apoe $e^{--}$macrophages. When macrophages were exposed to the apoptotic stimuli, TNF- $\alpha$, and cycloheximide, in the presence of serum that contained S1P, S1pr2 ${ }^{+/+} \mathrm{Apoe}^{-/-}$ macrophages exhibited a much greater extent of caspase- 3 activation at 12 and 24 hours than S1pr $2^{-/-}$Apoe $^{-/-}$macrophages (Sup- plemental Figure 7B). Anti-cleaved caspase-3 staining of the atherosclerotic lesions showed a higher number of apoptotic cells in

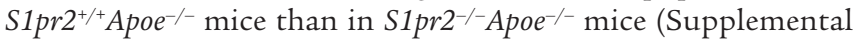
Figure 7C). Thus, S1pr2 $2^{-/-} A \mathrm{poe}^{-/-}$macrophages are more resistant to apoptosis compared with $S 1 p r 2^{+/+} A p o e^{-/-}$macrophages.

The activity of the Rho/ROCK/NF-KB pathway is inbibited in S1pr2deficient macrophages. S1PR2 is coupled to the $\mathrm{G}_{12 / 13} / \mathrm{Rho} / \mathrm{ROCK}$ pathway (28). Previous reports $(29,30)$ demonstrated that ROCK is involved in the activation of NF- $\kappa \mathrm{B}$, which plays a central role in inflammatory processes. In serum-deprived S1pr2 ${ }^{+/+} \mathrm{Apoe}^{-/-}$macrophages, S1P induced an increase in the amount of GTP-bound RhoA whereas S1P failed to stimulate RhoA in S1pr2 $2^{-/}$Apoe ${ }^{-/-}$macrophages (Figure 6A). Consistent with this, S1P stimulated phosphorylation of the ROCK substrate, MYPT1, at $\mathrm{Thr}^{850}$ in a manner sensitive to the ROCK inhibitor Y-27632 in S1 pr2 ${ }^{+/+} \mathrm{Apoe}^{-/-}$macrophages, whereas S1P did not in S1pr2 $2^{-/-}$Apoe $^{-/-}$macrophages (Figure 6B). $\mathrm{S} 1 \mathrm{P}$ also stimulated nuclear translocation of NF-кB (p65RelA), a reduction in the cytosolic IK-B $\alpha$, and phosphorylation at $\mathrm{Ser}^{536}$ of p65RelA in S1pr2 ${ }^{+/+} \mathrm{Apoe}^{-/-}$macrophages (Figure 6C and Supple- 
A
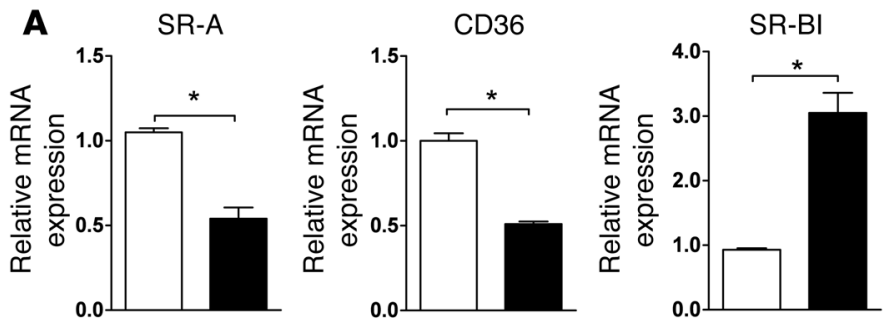

D
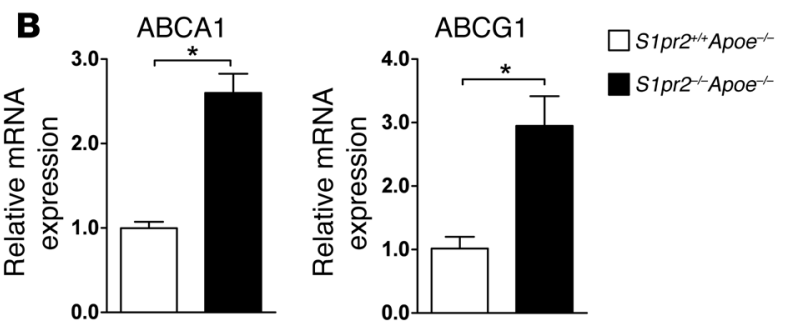

C

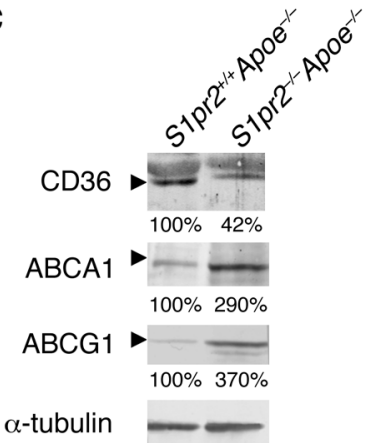

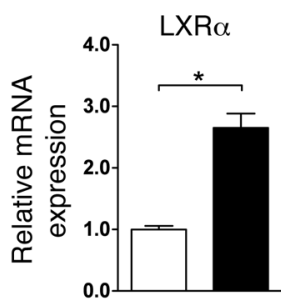
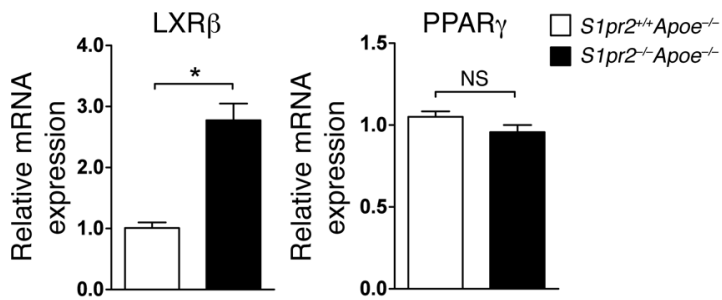

Figure 5

The effect of S1PR2 deficiency on the mRNA and protein expression of genes involved in modified LDL uptake and cholesterol efflux in mac-

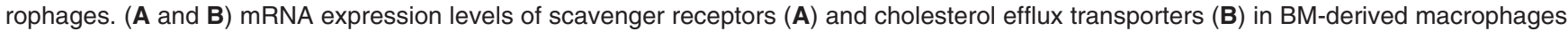
from S1 pr2 ${ }^{+/+} \mathrm{Apoe}^{-/-}$(white bars) and S1 pr2 ${ }^{-/-} \mathrm{Apoe}^{-/-}$(black bars) mice. (C) Western blot analysis of the protein expression of CD36, ABCA1, and ABCG1 from S1 pr2 $2^{++} \mathrm{Apoe}^{-/-}$and S1 pr2 ${ }^{-/}$Apoe ${ }^{-/-}$macrophages. (D) mRNA expression levels of transcription factors regulating cholesterol transporter expression. (A, B, and D) mRNAs were determined by real-time PCR. 18S rRNA was used as an internal control. Data are expressed as the ratio of the values in S1pr2 ${ }^{-/-} A p o e^{-/-}$mice over S1pr2 ${ }^{+/+} A p o e^{-/-}$mice $\left(n=4\right.$ each) and are shown as mean \pm SEM. ${ }^{\star} P<0.05$.

mental Figure 8, A and B). S1P-induced NF-кB phosphorylation and nuclear translocation were abolished by either Y-27632 or the S1PR2 antagonist JTE-K1 which specifically inhibited S1PR2mediated signaling (Supplemental Figure 8C) (31). In contrast, S1P

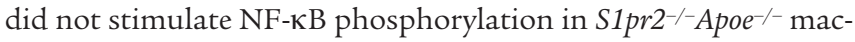
rophages. S1P stimulated the mRNA expression and the protein secretion of the NF- $\mathrm{KB}$ target gene TNF- $\alpha$ in S1pr2 ${ }^{+/+} \mathrm{Apoe}^{-/-}$macrophages, which was inhibited by either JTE-K1, Y-27632, or the NF-кB activation inhibitor BAY11-7085, whereas S1P did not stimulate TNF- $\alpha$ mRNA or protein expression in S1pr2 $2^{-/-}$Apoe $^{-/-}$macrophages (Figure 6D and Supplemental Figure 8D). These results together indicate that the S1PR2-coupled Rho/ROCK/NF-KB pathway mediates S1P stimulation of TNF- $\alpha$ expression.

Previous studies showed antiatherogenic actions of HDL, which is bound to S1P. We studied the effect of HDL on MYPT1 phosphorylation in macrophages. HDL at the final concentration, which contained the equivalent amount of S1P to $0.1 \mu \mathrm{M}$ unbound S1P, induced an extent of MYPT1 phosphorylation at Thr ${ }^{850}$ comparable to that of unbound S1P in S1pr2 ${ }^{+/+} \mathrm{Apoe}^{-/-}$macrophages (Supplemental Figure 8E). This HDL effect was abolished by JTE-K1. Thus, HDL-bound S1P activated the proatherogenic receptor S1PR2, like unbound S1P.

Previous studies showed the involvement of the Rho/ROCK pathway in the negative regulation of mRNAs of ABCA1 and LXR $\alpha$ (32). In S1pr2 $2^{+/+}$Apoe ${ }^{-/-}$macrophages but not S1pr2 ${ }^{-/-}$Apoe $^{-/-}$macrophages, the addition of either JTE-K1 or Y-27632 induced upregulation of mRNAs of ABCA1 and LXR $\alpha$ to the levels in S1pr2-/-Apoe ${ }^{-/}$ macrophages in the presence of serum that contained S1P (Figure 6, F and G). In contrast, the upregulation of CD36 mRNA in $\mathrm{S} 1 \mathrm{pr}^{+/+} \mathrm{Apoe}^{-/-}$macrophages compared with S1pr2 ${ }^{-/-} \mathrm{Apoe}^{-/-}$mac- rophages was abolished by either JTE-K1, Y-27632, or BAY11-7085 in the presence of S1P (Figure 6E). BAY11-7085 also strongly suppressed CD36 mRNA expression in S1pr2 $2^{-/-}$Apoe $^{-/-}$macrophages, similarly to its effects on TNF- $\alpha$ mRNA expression. These observations suggest that stimuli other than S1P substantially contribute to activation of NF- $\mathrm{KB}$ under this experimental condition. Treatment of S1pr2 ${ }^{+/+}$Apoe $^{-/-}$macrophages with either JTE-K1, Y-27632, or BAY11-7085 reduced oxLDL accumulation (Figure $6 \mathrm{H}$ ), which was consistent with their effects on the expression of CD36 and ABCA1. These observations indicate that S1PR2-coupled ROCK pathway mediates upregulation of CD36 and downregulation of ABCA1, leading to promotion of foam cell formation.

$S 1 P$ acts as a chemorepellent for macrophages via S1PR2. We studied transwell migration of peritoneal macrophages in the presence of either the positive (S1P in the lower chamber) or negative (S1P in the upper chamber) S1P gradient (Figure 7, A and B). S1P alone in the upper or lower chamber did not stimulate or inhibit migration of $\mathrm{S}_{\mathrm{pr}} \mathrm{P}^{+/+} \mathrm{Apoe}^{-/-}$macrophages (Figure 7, A and B), probably because $S 1 \mathrm{pr}^{+/+} A \mathrm{poe}^{-/-}$macrophages expressed both the chemoattractant receptor S1PR1 (S1PR3) and the chemorepellent receptor S1PR2 (Supplemental Figure 5A). In S1pr2 $2^{-/-}$Apoe $e^{-/-}$macrophages, however, $\mathrm{S} 1 \mathrm{P}$ alone in the lower chamber but not in the upper chamber stimulated migration in a dose-dependent manner very likely via the chemoattractant $\mathrm{S} 1 \mathrm{P}$ receptors (Figure 7, A and B). MCP-1 is a potent chemokine that stimulates monocyte transmigration into the arterial subendothelium. As reported previously (33), MCP-1 alone in the lower chamber induced a typical transwell migration response in both $S 1 \mathrm{pr}^{+/+} \mathrm{Apoe}^{-/-}$(Supplemental Figure 8F) and S1pr2-/-Apoe $e^{-/-}$(data not shown) macrophages in a dose-dependent manner. When applying S1P together with MCP-1 

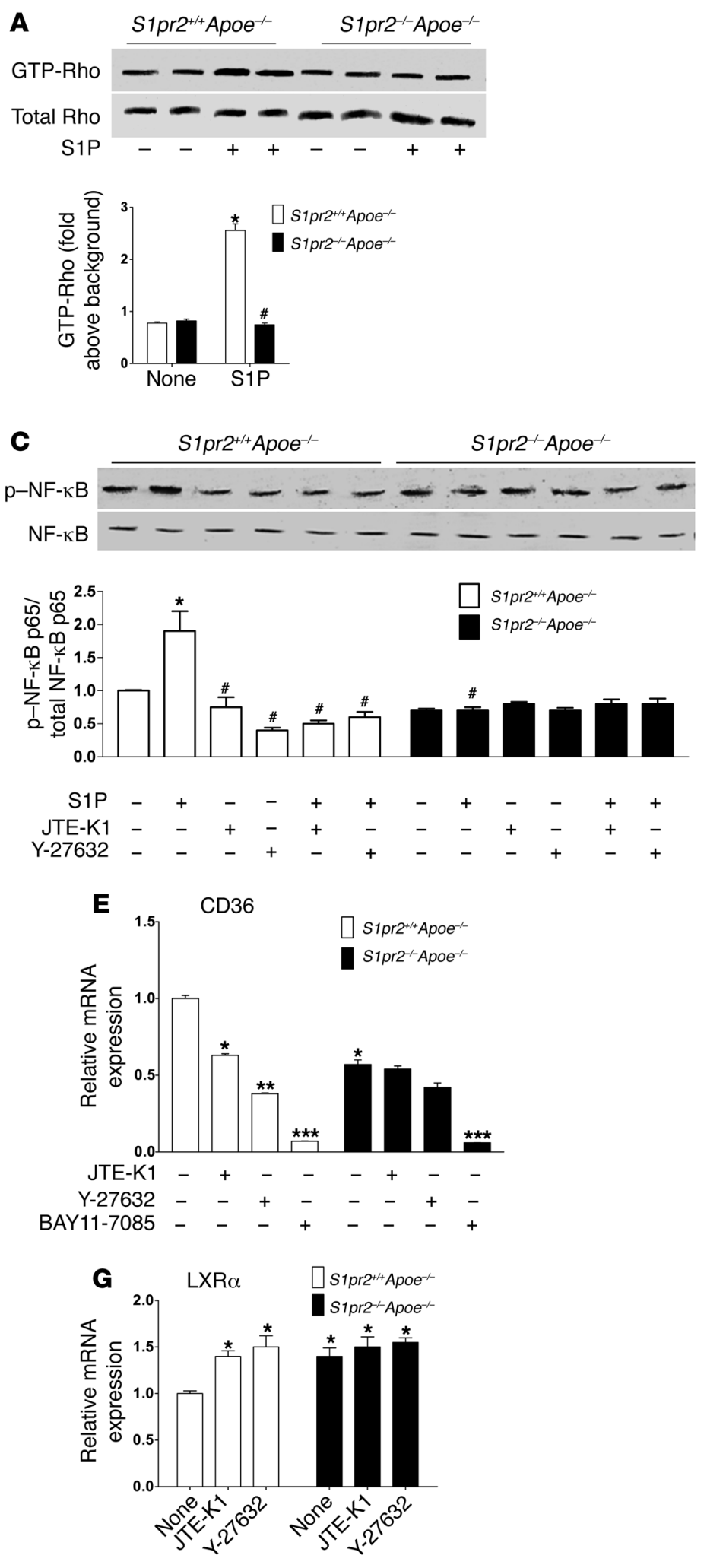
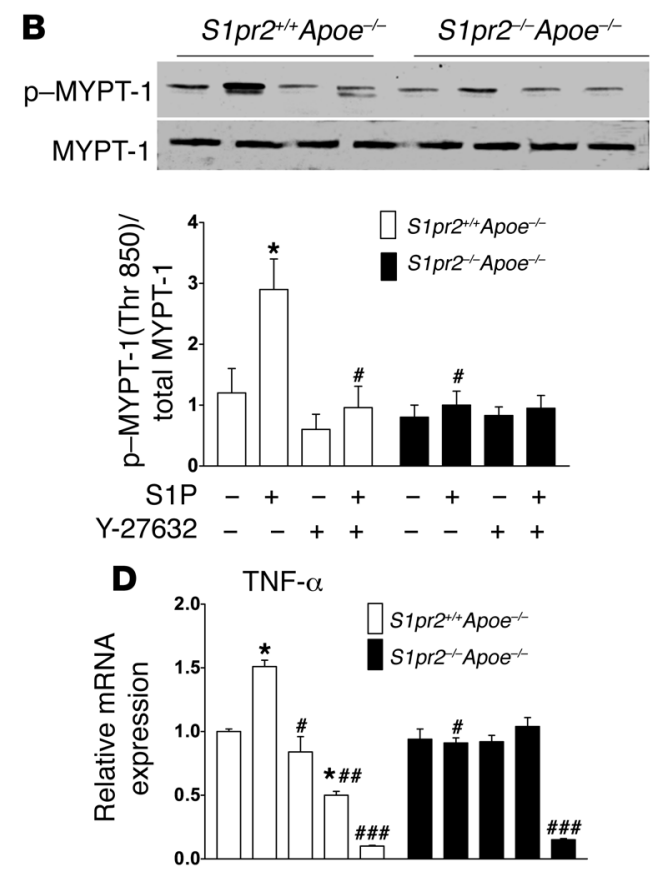

$\mathrm{S} 1 \mathrm{P}-++++-++++$

JTE-K1 - -+--++-

$\mathrm{Y}-27632---+---+$ BAY11-7085 - - - + - - - +
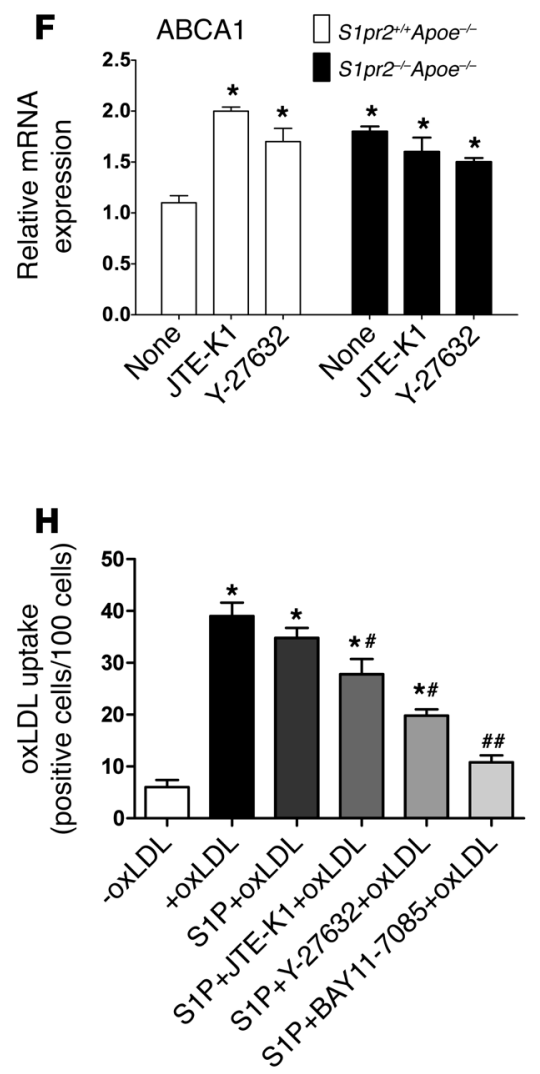


\section{Figure 6}

The activity of the Rho/ROCK/NF-KB pathway is inhibited in S1pr2deficient macrophages. (A-C) Serum-starved peritoneal macrophages from S1pr2 ${ }^{++} A$ poe $e^{-/}$(white bars) and S1pr2--Apoe ${ }^{-/-}$(black bars) mice were stimulated with $\operatorname{S1P}(0.1 \mu \mathrm{M})$ for 2 minutes $(\mathbf{A}), 5$ minutes (B), or 20 minutes (C) and analyzed for GTP-Rho (A), phosphorylation $\left(\mathrm{Thr}^{850}\right)$ of MYPT1 (B), and phosphorylation (Ser ${ }^{536}$ ) of NF-kB (p65) (C) as described in Methods ( $n=3$ each). (D) Peritoneal macrophages were stimulated with S1P $(0.1 \mu \mathrm{M})$ for 6 hours. TNF- $\alpha$ mRNA level was determined by real-time PCR. (E-G) BM-derived macrophages from S1 pr2 ${ }^{+/+} \mathrm{Apoe}^{-/-}$(white bars) and S1 pr2 ${ }^{-1-} \mathrm{Apoe}^{-/-}$(black bars) mice were cultured in DMEM containing 10\% FBS and treated with either JTE-K1, Y-27632 (Wako), or BAY11-7085 (Merck-Calbiochem) for 6 hours. mRNA expression levels of CD36 (E), ABCA1 (F), and LXR $\alpha(\mathbf{G})$ were determined by real-time PCR ( $n=5$ each). $(\mathbf{H})$ Foam cell formation assay. Serum-starved BM-derived macrophages from S1 pr2 ${ }^{+/+} \mathrm{Apoe}^{-/-}$mice were exposed to oxLDL for 6 hours. S1P $(0.1 \mu \mathrm{M})$, JTE-K1, Y-27632, and/or BAY11-7085 were added 6 hours before adding oxLDL. Quantified data of oil red O-positive cells are shown ( $n=3$ each). In A-H, the inhibitors ( $10 \mu \mathrm{M}$ JTE-K1, $10 \mu \mathrm{M}$ Y-27632, and $10 \mu \mathrm{M} B A Y 11-7085)$ were added $10-30$ minutes before S1P addition and present in the medium throughout the subsequent incubation. ${ }^{*} P<0.05 ;{ }^{* *} P<0.01 ;{ }^{* *} P<0.001$, as compared with nontreated S1pr2 ${ }^{+/+}$Apoe - $^{-}$macrophages. ${ }^{\#} P<0.05 ;{ }^{\# \#} P<0.01$; ${ }^{\# \# \#} P<0.001$, as compared with S1P-treated S1 pr2 ${ }^{+/+} A p_{0 e^{-/}}$macrophages. Data are expressed as the ratio of the values in treated cells over nontreated cells and are shown as mean $\pm \operatorname{SEM}(n=5$ each $)$.

in the lower chamber (Figure 7A), S1pr2-/-Apoe ${ }^{-/-}$macrophages showed marked stimulation of transwell migration in an S1P concentration-dependent manner by the cooperation of S1PR1 and CCR2. In contrast, S1pr2 ${ }^{+/+} A p^{-/-}$macrophages rather showed suppression of migration in an S1P concentration-dependent manner, indicating that S1PR2 acts as a chemorepellent receptor for macrophages. On the other hand, when various concentrations of S1P were added to the upper chamber in the presence of MCP-1 in the lower chamber, S1pr2 ${ }^{+/+} A \mathrm{poe}^{-/-}$macrophages but not $S 1 p r 2^{-/-} A p^{-1-}$ macrophages showed robust transwell migration (Figure 7B). Since the S1P concentration in the blood is thought to be higher than that in the arterial wall tissue, the negative S1P gradient illustrated as in Figure 7B seems to be mimicking the in vivo situation. Therefore, these results suggest that MCP-1-directed transmigration of circulating S1pr2 ${ }^{-/-} \mathrm{Apoe}^{-/-}$monocytes into the arterial subendothelium in the presence of negative S1P gradient is reduced compared with $S 1 p r 2^{+/+} A p o e^{-/-}$monocytes.

We determined cellular Rac activity, which plays a critical role in cell migration $(10,28)$, in macrophages (Figure $7 C)$. The unstimu-

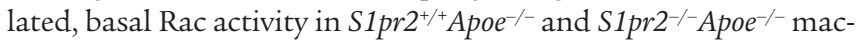
rophages was similar. MCP-1 stimulated Rac in both $S 1 \mathrm{pr}^{+/+} \mathrm{Apoe}^{-/-}$ and S1pr2 ${ }^{-1-} \mathrm{Apoe}^{-/-}$macrophages to a comparable extent. However, S1P stimulated Rac in S1pr2 $2^{-/-}$Apoe $e^{-/}$macrophages but not in S1pr2 ${ }^{+/+} A p_{00} e^{-/}$macrophages. Notably, S1P abolished MCP-1induced Rac activation in $S 1 p r 2^{+/+} A p^{-/-}$macrophages, but not in S1pr2 ${ }^{-/-}$Apoe $^{-/-}$macrophages.

To further investigate the role of S1PR2 in monocyte/macrophage migration in vivo, we performed the macrophage homing assay using GFP-expressing macrophages isolated from S1pr $2^{+/+} \mathrm{GFP}-\mathrm{Tg}$ and S1pr2-/-GFP-Tg mice. The GFP-expressing macrophages were injected via the tail vein into $S 1 p r 2^{+/+} A_{p o e^{-/-}}$recipient mice fed $\mathrm{HCD}$. Forty-eight hours later, we isolated the aorta and determined GFP-expressing macrophages that had infiltrated into the subendothelium, by using confocal microscopy. The higher number of
S1 $\mathrm{pr}^{+/+} \mathrm{GFP}-\mathrm{Tg}$ macrophages was found to infiltrate into the arterial wall compared with the injection of S1 $12^{-1-}$ GFP-Tg macrophages (Figure 7E). The protein expression levels of the integrins VLA-4 and LFA-1, the ligands of which are VCAM-1 and ICAM- 1 on the endothelium, and the major migratory chemokine receptor CCR2 were similar between $S 1 p r 2^{+/+}$and S1pr2 $2^{-/-}$macrophages (Figure 7D). These observations together suggest that S1PR2 deficiency in macrophages inhibits their transendothelial migration most likely through a direct action of S1P/S1PR2 on cell migration rather than secondary changes in other surface molecules involved in the transendothelial migration in S1pr2 ${ }^{-/-}$Apoe $e^{-/}$mice.

S1P affects leukocyte mobilization through S1PR1 and other $\mathrm{S} 1 \mathrm{P}$ receptors. We therefore examined the number of circulating blood monocytes. After 8 weeks on the HCD diet, S1pr2 ${ }^{+/+} \mathrm{Apoe}^{-/-}$ and S1pr2 $2^{-/-} A p_{0 e^{-/-}}$mice had similar numbers of total circulating monocytes as well as lymphocytes and neutrophils (Supplemental Table 4). The CD $11 \mathrm{~b}^{+}$Ly $6 \mathrm{C}^{\text {hi }}$ monocyte subset is shown to be preferentially recruited to atherosclerotic lesions (34). The number of circulating CD $11 \mathrm{~b}^{+} \mathrm{Ly} 6 \mathrm{C}^{\mathrm{hi}}$ monocytes was similar between S1pr2 ${ }^{+/+} \mathrm{Apoe}^{-/-}$and S1pr2 ${ }^{-/-}$Apoe ${ }^{-/-}$mice (Supplemental Figure 9).

The activity of the Rho/ROCK/NF-KB pathway is inhibited and Akt is enhanced in S1pr2-deficient ECs. EC dysfunction is generally accepted as the first step in atherosclerosis (35) and the Rho/ROCK pathway is implicated in EC dysfunction $(29,30,36)$. Mouse lung ECs (MLECs) from S1 pr2 ${ }^{+/+} \mathrm{Apoe}^{-/-}$mice expressed mRNAs of S1PR1, S1PR2, and S1PR3 (Supplemental Figure 10A). MLECs from S1pr2-/-Apoe - $^{--}$ mice expressed levels of S1PR1 and S1PR3 similar to those of S1pr2 ${ }^{+/+}$Apoe $^{-/-}$MLECs, but lacked S1PR2. S1P stimulated MYPT-1 phosphorylation in S1pr2 ${ }^{+/+} A p^{-/-}$MLECs and, to a lesser extent, in S1pr2-/-Apoe - $^{-1}$ MLECs, the latter being likely mediated by S1PR3. Y-27632 abolished S1P-induced stimulation of MYPT-1 phosphorylation in S1pr2 $2^{+/+} \mathrm{Apoe}^{-/-}$and S1pr2 ${ }^{-/-}$Apoe ${ }^{-/-}$MLECs (Figure 8, A and B).

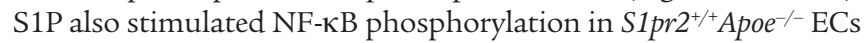
in a Y-27632-sensitive manner, but not in S1pr2-/-Apoe-/- MLECs (Figure 8, A and C). In contrast, S1P decreased Akt phosphorylation in a Y-27632-sensitive manner in S1pr2 ${ }^{+/+}$Apoe $^{-/-}$ECs, whereas S1P stimulated Akt phosphorylation in a Y-27632-insensitive manner in S1pr2 $2^{-/-}$Apoe $e^{-/-}$MLECs (Figure 8, A and D). Consistent with the S1P effects on Akt phosphorylation, S1P stimulated phosphorylation of eNOS at Ser ${ }^{1177}$, which is an Akt phosphorylation site, in S1pr2 ${ }^{-/-} \mathrm{Apoe}^{-/-}$ECs but reduced it in S1pr2 ${ }^{+/+} \mathrm{Apoe}^{-/-} \mathrm{ECs}$ (Figure 8, A and $\mathrm{E})$. Consistent with the results on NF- $\mathrm{KB}$ phosphorylation, S1P stimulated the mRNA expression of MCP-1 and GM-CSF, which

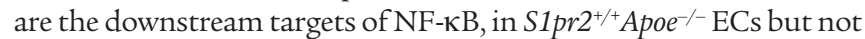
in S1pr2 $2^{--}$Apoe $e^{-/}$ECs (Figure 8, F and G). The basal and S1P-stimulated release of MCP-1 protein in S1pr2 $2^{-/-} \mathrm{Apoe}^{-/-}$ECs was reduced compared with S1pr2 $2^{++}$Apoe ${ }^{-/}$ECs (Supplemental Figure 10C).

S1P acts as a chemorepellent for SMCs and inbibits SMC proliferation via S1PR2. SMCs from S1pr2 ${ }^{+/+} A p o e^{-/-}$mice expressed S1PR1, S1PR2, and S1PR3 (Supplemental Figure 10B). SMCs from S1pr2 ${ }^{-/-} \mathrm{Apoe}^{-/-}$mice expressed levels of S1PR1 and S1PR3 similar to those from S1pr2 ${ }^{+/+}$Apoe $e^{-/-}$mice, but lacked S1PR2. S1pr2-/-Apoe $e^{-/-}$ SMCs showed more robust outgrowth from the aortic explants compared with S1pr2 $2^{+/+} \mathrm{Apoe}^{-/-}$SMCs (Figure 9A). The increased number of BrdU-positive SMCs was observed in the outgrowth from the aortic explants of S1pr $2^{-/-} A p o e^{-/-}$mice compared with $S 1 \mathrm{pr}^{+/+} \mathrm{Apoe}^{-/-}$mice (Figure 9A). In the transwell migration assay with $\mathrm{S} 1 \mathrm{P}$ in the lower chamber, S1pr2 ${ }^{+/+} A p o e^{-/-}$SMCs showed inhibition of both random migration and PDGF-directed migration in a S1P concentration-dependent manner, whereas S1pr2--Apoe $e^{-/}$SMCs did 

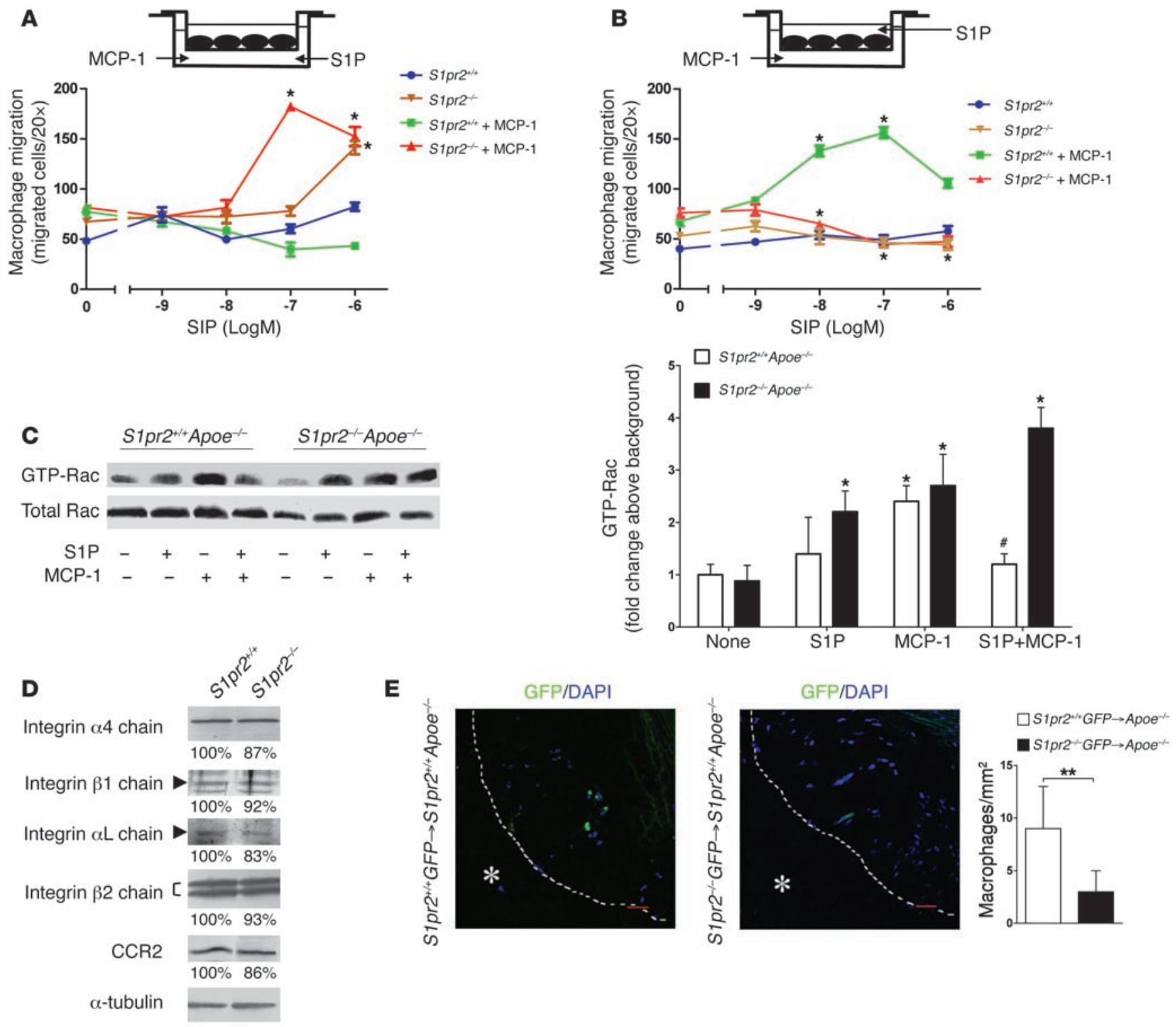

\section{Figure 7}

S1PR2 mediates migration inhibition in macrophages. (A and B) Effects of S1P and MCP-1 on transwell migration in serum-starved peritoneal macrophages isolated from S1 pr2 ${ }^{+/+} \mathrm{Apoe}^{-/-}$and S1 pr2 $2^{--} A p o e^{-/-}$mice. Macrophages were placed in the upper chamber. Various concentrations of S1P were added to either the lower chamber $(\mathbf{A})$ or the upper chamber $(\mathbf{B})$ in the presence or absence of MCP-1 $(100 \mathrm{ng} / \mathrm{ml})$ in the lower chamber. ${ }^{*} P<0.05$ compared with the value in the absence of S1P $(n=4$ each). (C) Effects of S1P and MCP-1 on Rac activity. Peritoneal macrophages were not treated or treated with either S1P $(0.1 \mu \mathrm{M})$ alone for 10 minutes, MCP-1 alone $(100 \mathrm{ng} / \mathrm{ml})$ for $3 \mathrm{minutes}$, or the combination of S1P and MCP-1. In the combination treatment, S1P was added 7 minutes before the addition of MCP-1. Amounts of GTP-bound Rac were determined by the pulldown assay $\left(n=3\right.$ each). ${ }^{*} P<0.05$, compared with nontreated macrophages; ${ }^{*}<<0.05$, compared with MCP-1-treated macrophages. (D) Western blot analysis of VLA-4 (integrin $\alpha_{4} \beta_{1}$ ), LFA-1 (integrin $\alpha_{L} \beta_{2}$ ), and CCR2 expression on peritoneal macrophages, with $\alpha$-tubulin as internal control. (E) Macrophage homing assay. Peritoneal macrophages isolated from S1pr2 ${ }^{+/+} \mathrm{GFP}-\mathrm{Tg}$ and $S 1 \mathrm{pr} 2^{-/-G F P-T g}$ mice were injected via the tail vein into S1pr2 ${ }^{++}{ }^{+} \mathrm{Poe}^{-/}$mice fed HCD $(n=3$ each). Sections of the aortic sinus were made and GFP-expressing cells infiltrating into the intima were observed under a fluorescent microscope (asterisks show the lumen), and GFP-positive cells were counted. Sections were counterstained with DAPI. Representative images (left) and quantified data are shown (right). ${ }^{* *} P<0.01$. Scale bars: $50 \mu \mathrm{m}$. Data are expressed as mean \pm SEM.

not show migration inhibition but rather S1P concentration-dependent stimulation of migration in the absence of PDGF (Figure 9B). In $S 1 p r 2^{+/+} A p o e^{-/-}$SMCs, S1P alone did not change the amount of GTP-Rac but inhibited PDGF-induced Rac activation, whereas S1P alone stimulated Rac in S1pr $2^{-1-}$ Apoe $e^{-/}$SMCs (Figure 9C).
Pharmacological blockade of S1PR2 inhibits atherosclerosis. Finally, we tested the effects of pharmacological S1PR2 blockade by systemic administration of the selective S1PR2 antagonist

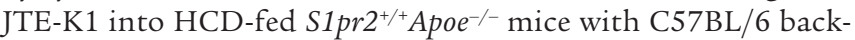
ground. Administration of JTE-K1 reduced the plaque area by 
A

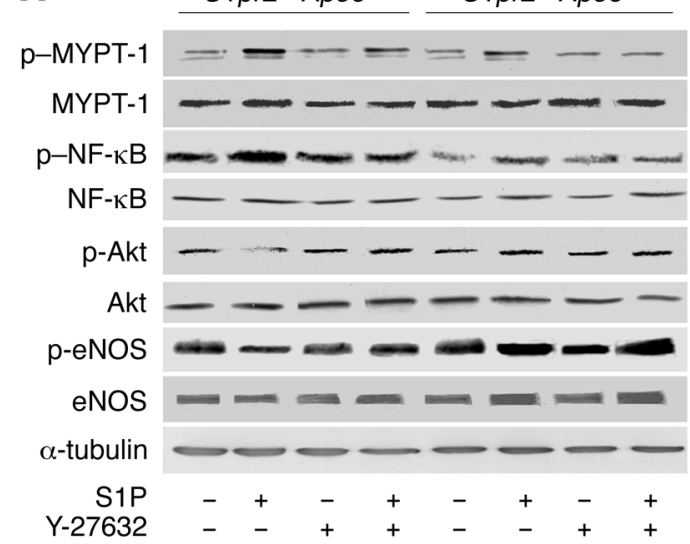

B

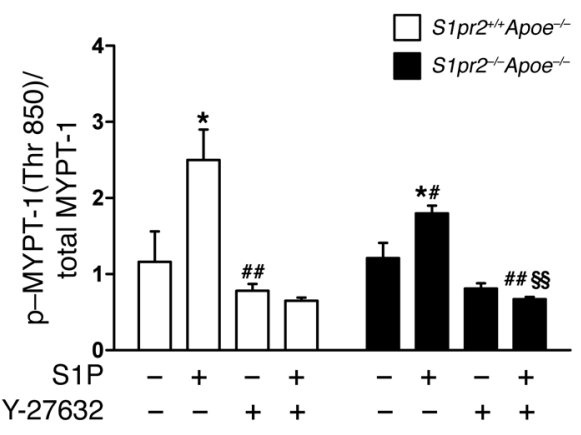

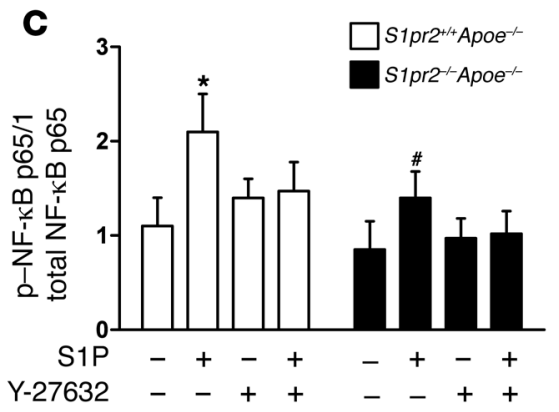

D
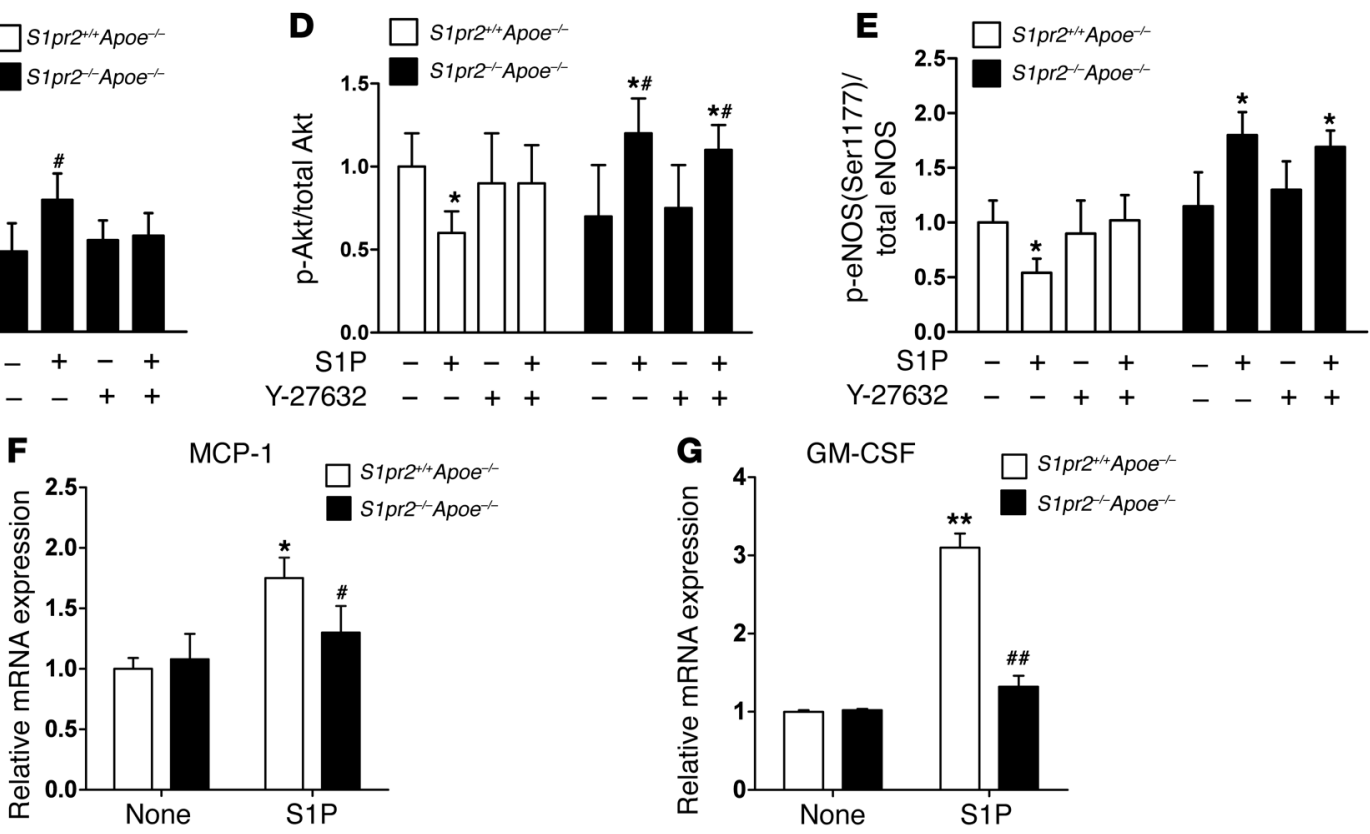

Figure 8

S1PR2 deficiency inhibits the activity of the Rho/ROCK/NF-kB pathway but stimulates those of Akt and eNOS in ECs. (A) Phosphorylation of MYPT1 at Thr850, NF-kB (p65) at Ser536, Akt at Ser473, and eNOS at Ser1177. MLECs isolated from S1pr2 ${ }^{+/+}$Apoe ${ }^{-/-}$and S1pr2 ${ }^{-l-} \mathrm{Apoe}^{-/-}$mice were serum starved for 15 hours and then stimulated with S1P $(0.1 \mu \mathrm{M})$ for 5 minutes (for MYPT1), for 20 minutes (for NF-kB), and for 20 minutes (for Akt and eNOS). Y-27632 (10 $\mu \mathrm{M})$ was added 30 minutes before the addition of S1P and included in the medium throughout subsequent incubations. Quantified data of phosphorylation of MYPT1 (B), NF-kB (C), Akt (D), and eNOS (E) in MLECs from S1pr2 ${ }^{+/+} \mathrm{Apoe}^{-/-}$(white bars) and S1pr2 ${ }^{-/-} \mathrm{Apoe}^{-/-}$(black bars) mice are shown ( $n=3$ each). (F and $\left.\mathbf{G}\right)$ MLECs from both groups of mice were serum starved for 15 hours and then stimulated with S1P $(0.1 \mu \mathrm{M})$ for 6 hours. mRNA expression levels of MCP-1 (F) and GM-CSF (G) were determined by real-time PCR. 18S rRNA was used as an internal control $\left(n=5\right.$ each). ${ }^{*} P<0.05$; ${ }^{* *} P<0.01$ compared with nontreated S1pr2 ${ }^{+/+}$Apoe ${ }^{-/-}$MLECs. ${ }^{\#} P<0.05$; \# $P<0.01$ compared with S1P-treated S1 pr2 ${ }^{+/+} A p o e^{-/-}$MLECs. $\$ \$ P<0.01$ compared with S1P-treated S1pr2 ${ }^{-/}$Apoe $^{-/}$MLECs. Data are expressed as mean \pm SEM.

approximately 60\% (Figure 10, A and B). Administration of the S1PR2 inhibitor resulted in the lower density of macrophages and higher density of SMCs in atherosclerotic lesions compared with vehicle control (Figure 10, C and D). These results indicate that pharmacological inhibition of S1PR2 successfully inhibits the atherosclerotic lesion formation. Moreover, we studied the effects of JTE-K1 on DiI-acLDL uptake and cholesterol efflux in macrophages. Pretreatment of S1pr $2^{+/+} A_{p o e^{-/-}}$macrophages with JTE-K1 suppressed uptake of DiI-acLDL and stimulated cholesterol efflux (Figure 10, E and F), thus mimicking the effects of S1pr2 gene disruption (Figure 4, C-E).

\section{Discussion}

Atherosclerosis is a chronic inflammatory disease in which multiple cell types are involved with complex interactions with each other $(1,2)$. Available evidence suggests that S1P has both proand antiatherosclerotic properties, and it is still debatable whether $\mathrm{S} 1 \mathrm{P}$ promotes or inhibits atherosclerosis $(13,37)$. It is unknown which cell type and which $\mathrm{S} 1 \mathrm{P}$ receptor mainly mediate the effects of S1P and its analogue FTY720 on atherosclerosis. In the present study, we focused on the role of S1PR2 in atherosclerosis because S1PR2 is expressed in all monocytes/macrophages, ECs, and SMCs and possesses a unique antimigratory activity. Our data provide 
A Explant tissues
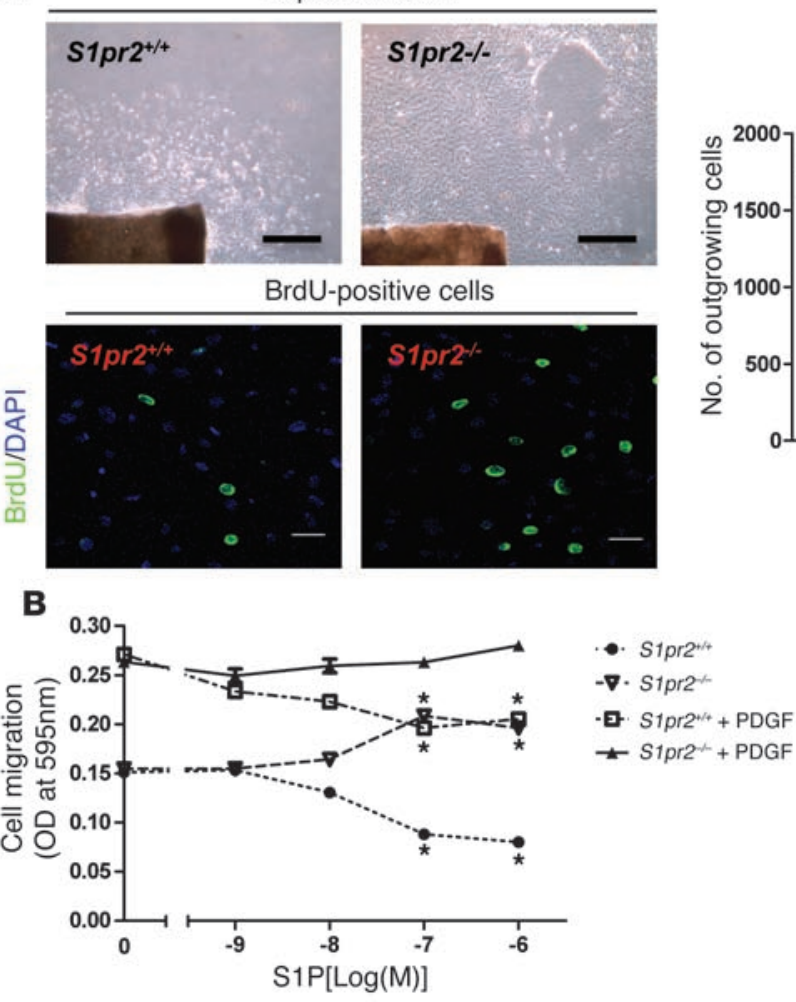
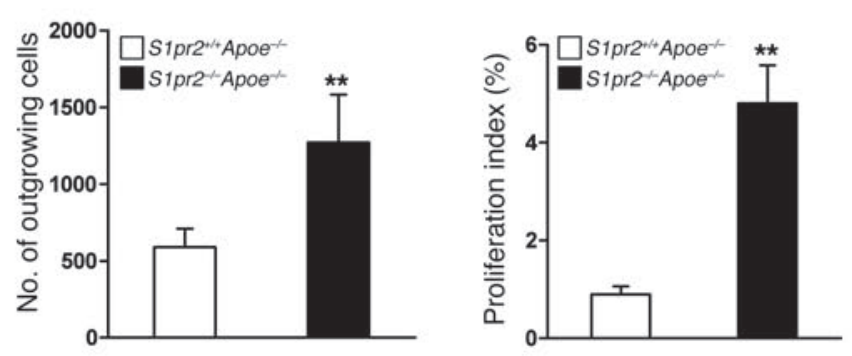

C

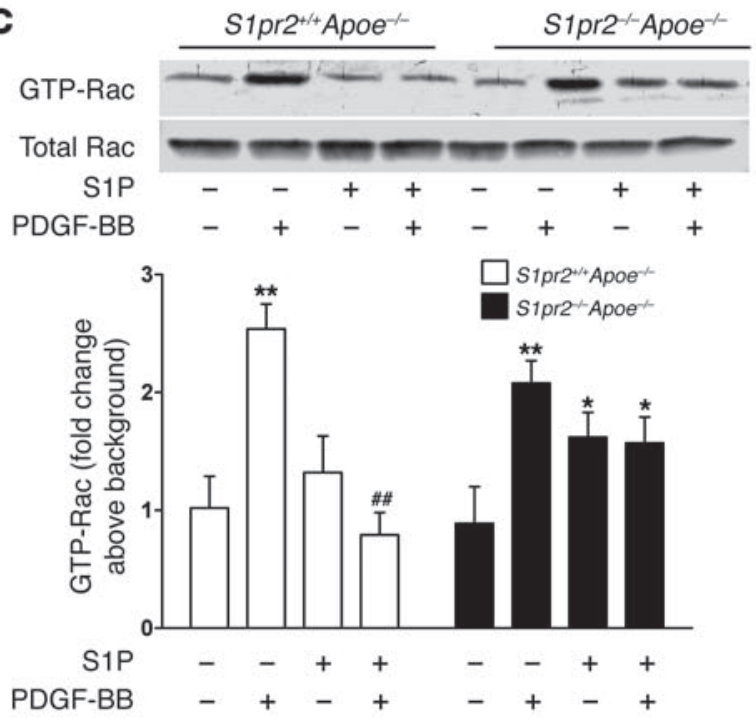

Figure 9

S1PR2 mediates S1P-induced chemorepulsion and inhibition of proliferation in SMCs. (A) Enhancement of SMC growth from aortic explants from

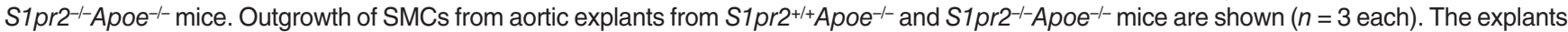
were individually placed in the culture dish and cultured for 4 days. (A) Phase contrast microscopic views (upper left) and quantified data of outgrowing cells are shown (upper right). Anti-BrdU immunostaining of proliferating cells. Outgrowing SMCs were labeled with BrdU and processed for anti-BrdU immunofluorescence staining. Fluorescent microscopic views (lower left) and quantified data of proliferating cells are shown (lower right). ${ }^{\star \star} P<0.01$. Scale bars: $100 \mu \mathrm{m}$ (upper panels); $20 \mu \mathrm{m}$ (lower panels). (B) Effects of S1P and PDGF on transwell migration of SMCs isolated from S1 pr2 ${ }^{+/+} \mathrm{Apoe}^{-/-}$and S1 pr2 $2^{-/} A \mathrm{poe}^{-/-}$mice. Various concentrations of S1P were added to the lower chamber in the presence and absence of PDGF-BB (10 ng/ml) in the lower chamber ( $n=4$ each). ${ }^{*} P<0.05$, compared with the values in the absence of S1P. (C) Effects of S1P and PDGF on Rac activity. SMCs isolated from S1 pr2 ${ }^{+/+} \mathrm{Apoe}^{-/-}$(white bars) and S1 pr2 ${ }^{-/-} \mathrm{Apoe}^{-/-}$(black bars) mice were not treated or treated with either S1P $(0.1 \mu \mathrm{M})$ alone for 5 minutes, PDGF-BB $(10 \mathrm{ng} / \mathrm{ml})$ alone for 3 minutes, or the combination of S1P and PDGF-BB. In the combination treatment, S1P was added 2 minutes before the addition of PDGF. Amounts of GTP-bound Rac were determined by the pulldown assay ( $n=3$ each). ${ }^{*}<0.05$; ${ }^{* *} P<0.01$ compared with nontreated SMCs. ${ }^{\# \# P}<0.01$ compared with PDGF-BB-treated SMCs. Data are expressed as mean \pm SEM.

evidence that S1PR2 exerts stimulatory effects on atherosclerosis, which are mediated through distinct actions in these multiple cell types with the major role of S1PR2 in monocytes/macrophages. Furthermore, our observations indicate usefulness of pharmacological blockade of S1PR2 in the inhibition of atherosclerosis.

BM chimera experiments in the present study indicate that S1PR2 in BM-derived cells plays the major promoting role in atherosclerosis. Circulating monocytes and monocyte-derived macrophages play a critical role in atherosclerosis. Our data show that S1PR2 in monocytes/macrophages promotes atherosclerosis through a multitude of mechanisms including the regulation of modified LDL accu- mulation, cytokine production, and cell migration. First, S1PR2 deficiency in macrophages markedly inhibits accumulation of modified LDL through both substantial decrease in uptake of modified LDLs and modest increase in cholesterol efflux, resulting in inhibition of oxLDL accumulation (Figure 4). These effects of S1PR2 deficiency lead to inhibition of foam cell formation and reductions in atherosclerotic lesions (Figure 1). Second, we observed that S1PR2 couples dominantly to the proatherogenic signaling pathway Rho/ROCK (38), which leads to NF-kB activation in macrophages as in other cell types (Figure 6, A-C, and Supplemental Figure 7) $(29,39)$. Consistent with S1PR2-mediated NF-KB activation, S1P stimulated the expres- 
A

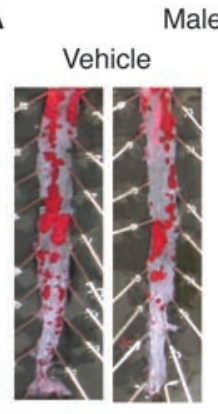

C

Vehicle
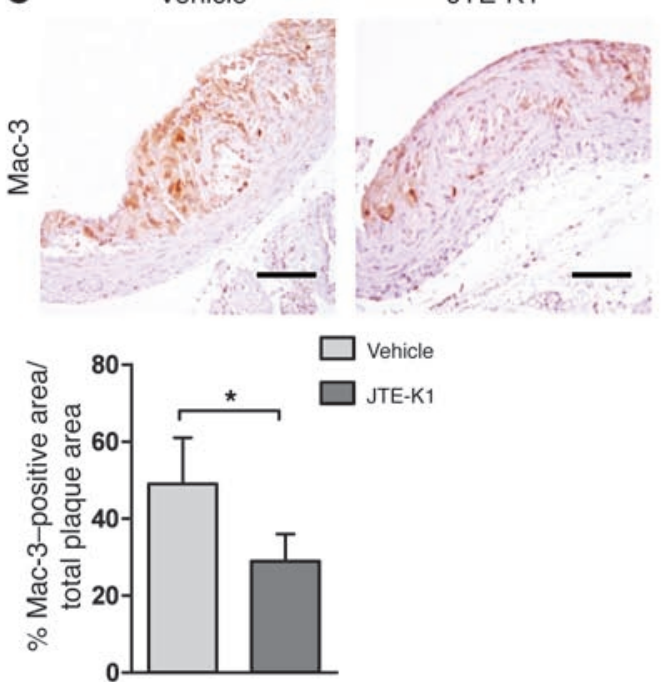

E
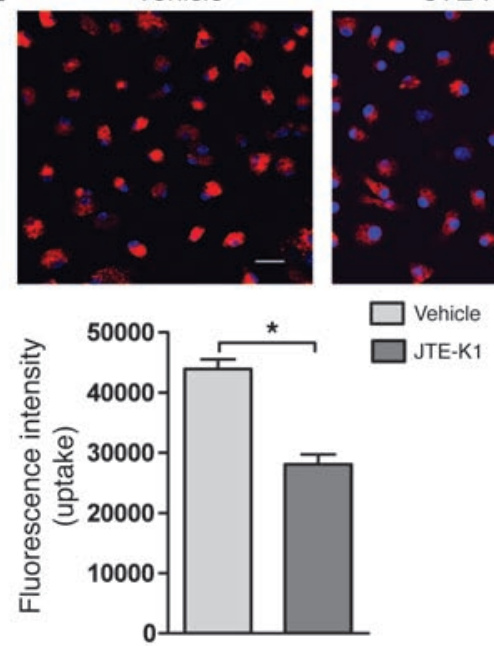

B

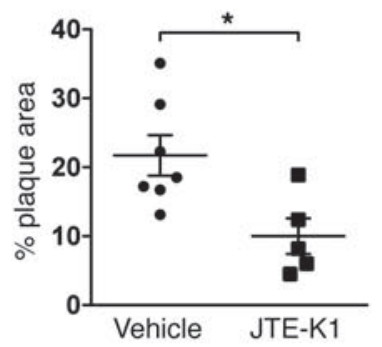

D Vehicle JTE-K1

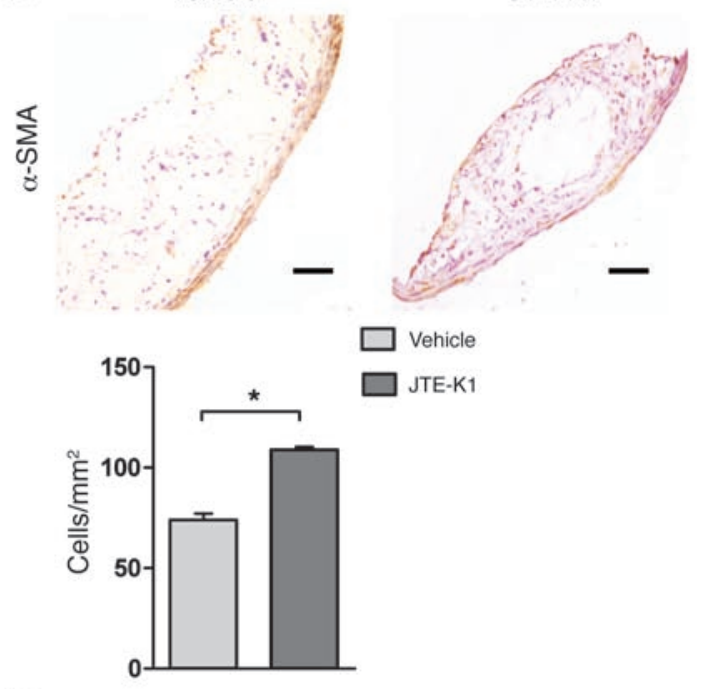

$\mathbf{F}$

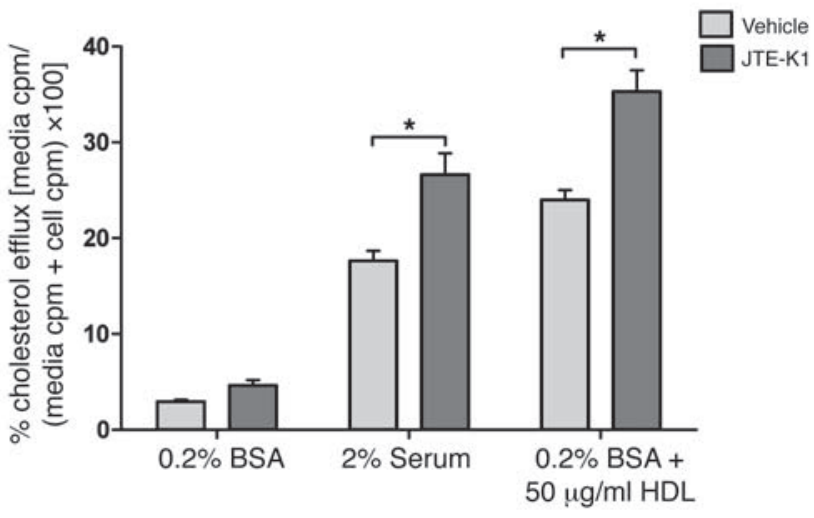

Figure 10

Pharmacological blockade of S1PR2 inhibits atherosclerosis. (A) S1PR2 antagonist JTE-K1 (25 mg/kg) was dosed to S1 pr2 $2^{++}$Apoe ${ }^{-/}$mice by oral gavage starting 2 weeks before 8 weeks of HCD. Representative oil red O staining of spread aortas from mice given vehicle $(n=7)$ or JTE-K1 $(n=5)$. (B) Quantified data of oil red O-stained plaque areas are shown. ${ }^{*} P<0.05$. (C and D) Immunostaining of Mac-3 (C) and $\alpha$-SMA (D) in the sections of the aortas from S1 pr2++A Apoe ${ }^{-/}$mice given vehicle or JTE-K1. Scale bars: 50 um. Quantified data are shown in the lower panels $\left(n=5\right.$ each). ${ }^{*} P<0.05$. (E) Uptake of Dil-labeled ac-LDL in JTE-K1 treated and nontreated S1 pr2 $2^{+/+} A p o e^{-/}$macrophages. Quantified data are shown (lower) $\left(n=3\right.$ each). Scale bars: $20 \mu \mathrm{m}$. ${ }^{*} P<0.05$. (F) Cholesterol efflux in JTE-K1-treated and nontreated S1 pr $2^{+/+}$Apoe $^{-/-}$macrophages $\left(n=3\right.$ each). ${ }^{*} P<0.05$. Data are expressed as mean \pm SEM. 
sion of the NF- $\kappa \mathrm{B}$ target gene TNF- $\alpha$ in a manner sensitive to the inhibitors of ROCK and NF-kB (Figure 6D). It is noted that S1PR2 deficiency induced upregulation of IL-10, a potent antiinflammatory cytokine, in the aorta and inhibited the phagocytotic activity of macrophages (Figure 2A and Supplemental Figure 6), showing that S1PR2 deficiency induced the changes of the phenotype and functional activities in macrophages. On the other hand, S1PR2 did not affect the differentiation of a circulating inflammatory monocyte $\left(\mathrm{CD} 11 \mathrm{~b}^{+}\right.$Ly6 $\mathrm{C}^{\mathrm{hi}}$ ) subset, which preferentially accumulates in plaques and becomes foam cells (Supplemental Figure 9) (34).

The downstream target molecules of the S1PR2/Rho/ROCK pathway in macrophages include the scavenger receptor CD36 and the cholesterol efflux ABC transporters (Figure 6, E and F). The mRNA expression of CD36 is upregulated by the S1PR2-coupled Rho/ ROCK/NF- $\mathrm{B}$ pathway, whereas the mRNA expression of the cholesterol efflux transporter ABCA1 is negatively regulated by S1PR2 and ROCK (Figure 6, E and F). The latter action of S1PR2 and ROCK seems to be at least in part mediated through downregulation of LXR expression (Figure 6G), which is a master regulator of cholesterol homeostasis and essential for transcriptional activation of $A B C A 1$ and $A B C G 1$ genes $(25,26)$. Eventually, the S1PR2-coupled Rho/ ROCK/NF- $\kappa B$ pathway in macrophages acts to stimulate inflammation and foam cell formation in atherosclerotic lesions (Figure $6 \mathrm{H}$ ).

Third, the chemorepellent receptor S1PR2 has a profound impact on transmigration of monocytes/macrophages to the arterial subendothelium. S1PR2 mediated inhibition of macrophage migration directed toward a chemoattractant in the presence of a positive S1P gradient (Figure 7, A and B), as in several different types of cells including SMCs, melanoma cells, and glioma cells $(10,40)$. In the in vitro transwell migration assay condition in which S1P was present in the upper chamber (negative S1P concentration gradient), which mimics a higher S1P concentration in the blood than in the subendothelial tissue, S1P substantially potentiated migration of S1pr2 ${ }^{+/+} \mathrm{Apoe}^{-/-}$macrophages toward MCP-1 in the lower chamber but not of S1pr2 ${ }^{-1-}$ Apoe $e^{--}$macrophages (Figure $7 \mathrm{~B}$ ). The results suggest that in the presence of a physiological S1P concentration gradient between the blood and the vascular wall, S1PR2 might promote macrophage transmigration into the subendothelium. In fact, we observed by the in vivo infusion experiments of GFPexpressing macrophages that S1PR2 possesses the stimulating role in transmigration of macrophages into the vascular wall (Figure 7E). The chemorepulsion of macrophages by S1PR2 is suggested to involve Rac inhibition and Akt inhibition (Figure 7C and Supplemental 7A). There is the possibility that the reduced migration of transferred S1pr2-deficient macrophages to the aortic intima could be secondary to changes in other macrophage surface molecules including integrins and chemokine receptors rather than reflecting a direct action of S1P/S1PR2 on transmigration. The expression of the major receptors for the adhesion molecules and the chemokine receptor CCR2 was similar between S1pr2 ${ }^{+/+}$Apoe $^{-/-}$and S1pr2 $2^{-/-}$Apoe $e^{-/-}$ macrophages (Figure 7D). However, it is not precluded that other surface molecules including selectins and chemokine receptors could be altered. Taken together, the stimulatory effects of S1PR2 on modified LDL accumulation, cytokine production, and transendothelial migration likely contribute to the proatherosclerotic role of macrophage S1PR2. Besides monocytes/macrophages, BM-derived mast cells, which also express S1PR2 to stimulate degranulation and are implicated in plaque progression and destabilization (41), may be involved in mediating the proatherogenic effect of S1PR2 because we observed a decrease in activated mast cells in the aortic wall of S1pr2-/-Apoe $e^{-/-}$mice (Figure $1 \mathrm{H}$ ). Elucidation of the role of mast cell S1PR2 in atherogenesis awaits further investigation.

Another substantial site of S1PR2 action in atherosclerosis may be ECs, which are the cells that release antiatherogenic mediator $\mathrm{NO}$ and proatherogenic cytokines, and mediate adhesion and transmigration of leukocytes. The NO-synthesizing enzyme eNOS and its product NO have atheroprotective properties (20). S1PR2 deficiency resulted in heightened phosphorylation of eNOS in the aortas in vivo (Figure 2D). Consistent with this, S1P induced stimulation of eNOS phosphorylation probably via S1PR3 (42) in isolated S1pr2 $2^{-/-} A p o e^{-/-}$ECs, whereas in S1pr2 ${ }^{+/+}$Apoe ${ }^{-/-}$ECs, S1P inhibited eNOS phosphorylation, which we found to be ROCK dependent (Figure 8, A and E). Most likely, S1P stimulation of eNOS phosphorylation in S1pr2-deficient ECs is mediated through the well-known eNOS-activating protein kinase Akt (43) because S1P stimulated Akt phosphorylation in S1pr2 ${ }^{-/-}$Apoe $^{-/-}$ECs but inhibited it in S1pr2 ${ }^{+/+} A_{p o e^{-/-}}$ECs in a ROCK-dependent manner (Figure 8A). The negative regulation of Akt phosphorylation by S1PR2 is likely mediated by ROCKdependent PTEN stimulation (9). Available evidence shows that the mechanisms for the atheroprotective actions of NO involve the inhibition of cytokine production, platelet aggregation and adhesion, leukocyte adhesion and activation, and smooth muscle proliferation (20). S1PR2 deficiency resulted in suppression of the expression of the proinflammatory cytokines including MCP-1 and GM-CSF in ECs as well as the aortas (Figure 2A, Figure 8, F and G, and Supplemental Figure 10C). MCP-1 is a powerful in vivo chemoattractant for monocytes/macrophages, as evidenced by the fact that MCP-1 deficiency markedly inhibits atherosclerosis with fewer macrophages in the aortic wall in Ldlr-null and Apoe-null mice $(44,45)$. GM-CSF was recently shown to be essential for proliferation of the intimal cells including dendritic cells in early atherosclerotic lesions (46). Thus, S1PR2 in ECs could participate in atherosclerosis by regulating adhesion molecule expression, cytokine production, and consequently monocyte/ macrophage flux, platelet activation, and thrombus formation, and intimal cell proliferation through Rho/ROCK/PTEN-mediated Akt/eNOS regulation and Rho/ROCK/NF- $\mathrm{KB}-$ mediated regulation of proinflammatory gene expression.

The present study suggests that S1PR2 in SMCs may destabilize plaques by decreasing the density of $\alpha$-SMA-positive SMCs, which is likely mediated through inhibition of proliferation and SMC migration into the intima (Figure 1F and Figure 9). However, the effects of S1PR2 on other SMC phenotypes, including matrix metalloprotease production and cytokine release, are still unknown. The relationship between macrophage apoptosis and atherogenesis is also complex (47). Decreased uptake of modified LDL and increased cholesterol efflux delay the accumulation of free cholesterol in macrophages, which is toxic to cells (27). We observed that, in contrast with S1pr2 ${ }^{+/+} \mathrm{Apoe}^{-/-}$macrophages in which S1P inhibited Akt phosphorylation, S1P stimulated Akt phosphorylation and inhibited caspase-3 activation in S1pr2 ${ }^{-/-}$Apoe $^{-/-}$macrophages (Supplemental Figure 7). These effects may affect macrophage survival and apoptosis and, as a result, the state of inflammation in the plaque $(47,48)$. Further study is required to define the effect of S1PR2 on plaque stability and its mechanism.

HDL, an atheroprotective lipoprotein, is the principal protein that is bound to S1P in plasma as well as albumin. The atheroprotective effects of HDL, including Akt-mediated eNOS stimulation 
and inhibition of E-selectin expression, were shown to be mediated via S1PR3 $(4,42)$. Our in vitro data showing that HDL stimulated MYPT1 phosphorylation in S1pr2 ${ }^{+/+}$macrophages via S1PR2 and ROCK (Supplemental Figure 8E) suggest that S1P that is bound to HDL can activate S1PR2 to stimulate the proinflammatory signaling pathway at least in vitro in macrophages. Therefore, it is possible that the S1PR2-activating capacity of HDL may reduce an atheroprotective activity of HDL.

Currently, most therapy for human atherosclerotic lesions is to reduce the concentration of LDL particles in the blood mainly by HMG CoA reductase inhibitors $(1,49)$. Statins inhibit the accumulation of modified LDL in the subendothelial layer mainly by lowering blood cholesterol concentration. Although statins also exert plaque stabilizing and antiinflammatory effects $(1,36,49)$, no therapy to directly target foam cell formation in the face of elevated circulating LDL is currently available. In genetic mouse models for atherosclerosis, pharmacological blockade of chemoattractant receptors that are expressed in monocytes, including leukotriene B4 receptor and RANTES receptors, effectively inhibited plaque formation $(50,51)$. In the present study, S1PR2 blockade using systemic administration of a selective S1PR2 blocker reproduced the phenotypes of S1pr2-deficient mice; it reduced plaque areas and macrophage infiltration in the aorta and inhibited modified LDL uptake and stimulated cholesterol efflux in macrophages (Figure 10). It is of note that S1PR2 deficiency affects various cell functions including migration, proliferation, apoptosis, cytokine production, and lipid accumulation in macrophages, ECs, and SMCs. Hence, clarification of differences in the effects between the above-mentioned chemoattractant receptor antagonists and the S1PR2 antagonist deserves further investigation. In conclusion, these observations provide strong support for S1PR2 as a promising novel target for the treatment of atherosclerosis.

\section{Methods}

Materials. DL-erythro-1P was purchased from BIOMOL. MCP-1, HDL, and oxLDL were prepared from human serum as described previously (52). The following antibodies were used: phospho-(Ser $\left.{ }^{473}\right)$-Akt (\#4060; Cell Signaling), total Akt (\#9272; Cell Signaling), phospho-(Thr $\left.{ }^{853}\right)$-MYPT1 (36-003; Upstate Biotechnology), total MYPT1 (PRB-457C; Covance), phospho-(Ser $\left.{ }^{536}\right)-N F-\kappa B$ (RelA/p65) (\#3031; Cell Signaling), total NF-кB (sc-8008; Santa Cruz Biotechnology Inc.), phospho-(Ser $\left.{ }^{1177}\right)$-eNOS (\#9571; Cell Signaling) and total eNOS (\#9586; Cell Signaling), phospho-p44/42 MAPK (Thr202/Tyr204) (\#4370; Cell Signaling), p44/42 MAPK (\#9102; Cell Signaling), CD36 (sc-9154; Santa Cruz Biotechnology Inc.), ABCA1 (NB400-164; Novus Biologicals), ABCG1 (AB36969; Abcam), integrin $\alpha 4$ chain (AB1924; Millipore), $\alpha$ L chain (ab25173; Abcam), $\beta 1$ chain (sc-8978; Santa Cruz Biotechnology Inc.), $\beta 2$ chain (557437; BD Biosciences Pharmingen), Histon H1 (sc-10806; Santa Cruz Biotechnology Inc.), and CCR2 (Epitomics). 1-[1,3-Dimethyl-4-(2-methylethyl)-1H-pyrazolo[3,4b]pyridine-6-yl]-4-(2,6-dichloro-4-pyridinyl)-semicarbazide (JTE-K1) was synthesized as described (31).

Animals. S1 pr2 $2^{-/}$mice and LacZ-knockin (S1pr2 $\left.2^{\text {LacZ/+}}\right)$ mice were previously described (12). S1 pr2 $2^{-/-}$mice and $S 1 p r 2^{\mathrm{LacZ} Z /+}$ mice that had been backcrossed to $\mathrm{C} 57 \mathrm{BL} / 6$ at indicated times were crossed with $A p o e^{-/-}$mice $(\mathrm{C} 57 \mathrm{BL} / 6$ background). Heterozygous mice (S1Pr2 $\left.{ }^{+/-} \mathrm{Apoe}^{+/-}, \mathrm{S} 1 \mathrm{pr} \mathrm{LacZ} /+^{+} \mathrm{Apoe^{+/- }}\right)$ were intercrossed to generate homozygous $A p o e^{-/-}$mice bearing combinations of $S 1 \mathrm{pr}^{+/+}, \mathrm{S} 1 \mathrm{pr}^{+/-}, \mathrm{S} 1 \mathrm{pr} 2^{-/-}$, and $\mathrm{S} 1 \mathrm{pr} 2^{\mathrm{LacZ} /+}$ mice. This process resulted in the backcrosses to C57BL/ 6 of up to 7 generations. GFP-Tg mice, which were kindly donated by Masaru Okabe (Osaka University, Suita, Japan), were housed in a temperature-controlled conventional facility $\left(24^{\circ} \mathrm{C}\right)$ under a 12-hour light/12-hour dark cycle with free access to regular chow or an HCD (1.25\% cholesterol, $7.5 \%$ cocoa butter, $7.5 \%$ casein, $0.5 \%$ cholate) (Ninox), which started at 6 weeks of age, and water. JTE-K1 $(25 \mathrm{mg} / \mathrm{kg}$ in $200 \mu \mathrm{l} /$ mouse, twice per day) suspended in $0.5 \%$ metolose (Shin-Etsu Chemical) was dosed to Apoe $e^{-/-}$mice by oral gavage starting 2 weeks before HCD. In parallel, control Apoe $e^{-/}$littermates received $0.5 \%$ metolose. All experiments using mice were approved by and performed according to the Guidelines for the Care and Use of Laboratory Animals of Kanazawa University, which strictly conforms to NIH guidelines.

Atherosclerotic lesion analysis. After the indicated time period of HCD feeding, mice were sacrificed with ether anesthesia and perfused with $4 \%$ PFA in $0.9 \%$ saline. The entire aorta from the root, extending 5 to $10 \mathrm{~mm}$ after bifurcation of the iliac arteries and including the subclavian, right, and left carotid arteries, was dissected and opened longitudinally. After staining with oil red $\mathrm{O}$, the aortas were scanned and the percentage plaque area over total aortic area was quantified using ImageJ (NIH) software. For histological and immunohistological analyses, the aortas were snap-frozen in OCT compound (Sakura FineTek). Serial cryostat sections of $10-\mu \mathrm{m}$ thickness were prepared. For immunohistochemistry and immunofluorescence, cryosections were incubated with primary antibodies against either Mac-3 (BD Biosciences), $\alpha$-SMA (Sigma-Aldrich), CD3 (DAKO), CD31 (553171, clone MEC13.3; BD Biosciences), VCAM-1 (BD Biosciences), ICAM-1 (BD Biosciences), or cleaved caspase-3 (Cell Signaling) followed by incubation with the secondary antibodies conjugated with horseradish peroxidase or Alexa Fluor 488 and nuclear counterstaining with hematoxylin or DAPI (Molecular Probes). The sections were observed with a microscope (BX41; Olympus) or confocal fluorescence microscope (LSM 510 Pascal; Carl Zeiss). Positive staining areas were quantified by using ImageJ (NIH) software.

Blood and plasma analysis lipid measurements. For blood cell counting with leukocyte differential analysis, blood was collected via vena cava from nonfasted mice. For plasma lipid analysis, mice were fasted for 16 hours before collecting blood. Plasma was separated by centrifugation and stored at $-80^{\circ} \mathrm{C}$ until further analysis. Plasma concentrations of total cholesterol and triglycerides were determined with autoanalyzer by SRL. An HPLC system with 2 tandem gel permeation columns was used to evaluate the size distribution of plasma lipoprotein particles (Skylight Biotech Inc.). Plasma $\mathrm{S} 1 \mathrm{P}$ concentration and HDL-associated S1P were determined by high-performance liquid chromatography as described previously (53).

Isolation and culture of circulating monocytes, peritoneal macrophages, and BMderived macrophages. Circulating monocytes were isolated by using Lymphoprep (Nycomed Pharma AS) density gradient centrifugation followed by magnetic separation using CD11b Microbeads mouse/human and MS columns (Miltenyi Biotec). Peritoneal macrophages were harvested from mice 3 days after intraperitoneal injection of thioglycollate $(2 \mathrm{ml}, 4 \%)$ and plated in DMEM containing $10 \%$ FBS. After 4 hours incubation at $37^{\circ} \mathrm{C}$, nonadherent cells were removed and adherent cells were used for the experiments. For the preparation of BM-derived macrophages, BM cells isolated from the femur and tibia were suspended in DMEM containing 10\% FBS. After treatment with red blood cell lysis buffer (Pharm Lyse; BD), cells were cultured with DMEM containing 10\% FBS and $5 \mathrm{ng} / \mathrm{ml} \mathrm{M-CSF} \mathrm{(Sigma-}$ Aldrich) for 10 days. Almost all adherent cells expressed the macrophagespecific markers, including Mac-3.

Isolation and culture of primary mouse lung endothelial cells and aortic SMCs. Mouse lung endothelial cells (MLECs) were isolated from 6-week-old mice by using digestion with collagenase A (Roche) magnetic separation using rat anti-mouse CD105 antibody (550546; BD Biosciences) and goat anti-rat antibody-conjugated magnetic beads, and MS columns (Miltenyi Biotec) as described previously (12). MLECs were cultured on type I collagen (Nitta Gelatin) coated plastic dishes, in EBM-2 medium (Lonza) containing growth factor supplements and $2 \% \mathrm{FBS}$, according to the instructions of the supplier. 
Mouse aortic SMCs were isolated by the explant method from 6-week-old mice, as described previously (10). In brief, aortas were removed, deendothelialized, and then cut open longitudinally. SMCs were cultured on type I collagen-coated dishes in Advanced DMEM supplemented with glutaMAX (Invitrogen) and 10\% FBS. Proliferation was evaluated with anti-BrdU (BD Biosciences - Pharmingen) immunofluorescence staining, in which SMCs were incubated with BrdU (a final concentration of $10 \mu \mathrm{M}$ ) (BD Biosciences - Pharmingen) for 30 minutes and then fixed in 4\% PFA in PBS (54).

Phagocytosis assay. To examine phagocytotic capacity of macrophages, we incubated peritoneal macrophages with fluorescent monodisperse polystyrene microspheres (Fluoresbrite Carboxylate Microspheres, $1.75 \mu \mathrm{m}$ in diameter; Polysciences Inc.) for 1 hour. After 100\% methanol fixation, cells were counterstained with DAPI; then positive cells were counted under a fluorescent microscope.

Determination of oxLDL uptake, binding and uptake of DiI-acLDL, and cholesterol efflux assay. To evaluate the ability of oxLDL uptake, macrophages were incubated with human oxLDL $(50 \mu \mathrm{g} / \mathrm{ml})$ for 6 hours at $37^{\circ} \mathrm{C}$, followed by staining with oil red $\mathrm{O}$ and counterstaining with hematoxylin. The number of oil red O-positive foam cells was counted under a microscope. To assess the intracellular uptake of Dil-acLDL (Harbor Bio-Products), macrophages were incubated for 4 hours at $37^{\circ} \mathrm{C}$. Fluorescence intensity was observed using a confocal fluorescence microscope and quantified using ImageJ software. Macrophages were loaded with ${ }^{3} \mathrm{H}$-cholesterol by incubating them in DMEM plus $10 \%$ FBS containing ${ }^{3} \mathrm{H}$-cholesterol $(2 \mu \mathrm{Ci} / \mathrm{ml})$ for 24 hours. Cholesterol efflux for 4 hours was determined in the presence of DMEM containing either $0.2 \%$ BSA (Sigma-Aldrich), $2 \% \mathrm{FBS}$, or $50 \mu \mathrm{g} / \mathrm{ml}$ HDL as acceptors. The cholesterol efflux was expressed as the percentage of the radioactivity released from the cells in the medium relative to the total radioactivity in the cells plus medium.

Transwell migration assay. Transwell migration of macrophages and SMCs was determined in a modified Boyden chamber (Neuroprobe) using polycarbonate filters with $8-\mu \mathrm{m}$ pores as described in detail previously (28). Peritoneal macrophages $\left(2 \times 10^{5}\right)$ or SMCs $\left(1 \times 10^{5}\right)$ in $200 \mu$ l of serum-free medium containing $0.1 \%$ fatty acid-free BSA with or without S1P were loaded into the upper wells, whereas the lower wells were filled with the same medium containing MCP-1 (Sigma-Aldrich) or PDGF-B chain (PeproTech). The cells were allowed to migrate across the porous filter for 12 hours (for macrophages) or 6 hours (for SMCs) at $37^{\circ} \mathrm{C}$, followed by counting the cells that migrated to the lower side of the filter under a microscope.

Macrophage homing assay. Macrophage homing assay was performed as described previously (55). Macrophages from GFP-Tg mice resuspended at $10^{7}$ cells $/ \mathrm{ml}$ in PBS plus $0.1 \%$ BSA $(200 \mu \mathrm{l})$ were injected into the tail vein of mice. 48 hours later, mice were sacrificed and perfused with $0.9 \%$ saline. Heart base and ascending aorta were frozen by embedding in OCT and cryosectioned at $15 \mu \mathrm{m}$. GFP-positive macrophages infiltrating into the atherosclerotic lesions were counted in 70 serial sections per mouse, spanning the proximal $1 \mathrm{~mm}$ of the aortic sinus region, by a confocal fluorescence microscope, and their total number was divided by the oil red $\mathrm{O}$-stained lesion area.

$B M$ transplantation. BM transplantation (BMT) was performed as described previously (12). Unfractionated BM cells $\left(2 \times 10^{6}\right.$ cells/recipient $)$ collected from S1pr2-/-Apoe ${ }^{-/}$and S1 $\mathrm{pr}^{+/+} \mathrm{Apoe}^{-/-}$mice were injected into recipient mice via the tail vein. After transplantation, all mice were fed a regular chow for 4 weeks before they were switched to HCD.

Flow cytometry. Mouse tail blood was collected with $5 \mathrm{mM}$ EDTA, treated with Fc Block (BD Biosciences), and stained with the following antibodies (15 minutes at $4^{\circ} \mathrm{C}$ in the dark): $\mathrm{CD} 45-\mathrm{APC}-\mathrm{Cy} 7$ (eBioscience), CD11b-
PE-Texas Red (Invitrogen), Gr-1-Pacific Blue (eBioscience), CD3-PerCyPCy5.5 (eBioscience), TER119-PerCyP-Cy5.5 (eBioscience), CD19-PerCyPCy5.5 (eBioscience), NK1.1-PerCyP-Cy5.5 (eBioscience), and Ly6C-APC (BioLegend). Red blood cells were then lysed with FACS lysing solution (BD Biosciences). Cells were resuspended in $200 \mu \mathrm{l}$ of FACS buffer (PBS containing $2 \% \mathrm{FBS}$ ) and analyzed by FACS Aria II (BD Biosciences). Data were transferred and reanalyzed with FlowJo software (Tree Star Inc.).

Determination of the activities of Rho and Rac. Pull-down assays to determine GTP-bound active forms of RhoA and Rac1 were performed as described in detail previously (10). Cell lysates were prepared and incubated with GST-Rhotekin (for determination of Rho activity) or GST-PAK1 (for determination of Rac activity), which were immobilized onto glutathione$S$-sepharose 4B beads (GE Healthcare), followed by Western blotting using specific monoclonal antibodies against RhoA (Santa Cruz Biotechnology Inc.) and Rac1 (Upstate Biotechnology).

Isolation of $m R N A$, RT-PCR, and quantitative real-time PCR. Total RNA was isolated from entire aortas, monocytes/macrophages, and ECs using TRIzOL (Invitrogen). RT-PCR was performed as described previously by using the reverse transcriptase ReverTra Ace and GoTaq Flexi DNA Polymerase. PCR products were separated on $2 \%$ agarose gels, visualized by ethidium bromide staining, and normalized for the expression levels of GAPDH mRNA. Sequences of specific primers and amplified product sizes are listed in Supplemental Table 5. Real-time quantitative PCR was performed using the ABI PRISM 7300 sequence detection system (Applied Biosystems), as described previously (12). Primers are listed in Supplemental Table 6. $\Delta$ Ct was calculated as (gene of interest $\mathrm{Ct}$ ) - (18s rRNA $\mathrm{Ct}$ ) using Sequence Detector (Applied Biosystems) and Microsoft Excel (Microsoft Corp.). The relative quantity of mRNA of gene of interest was

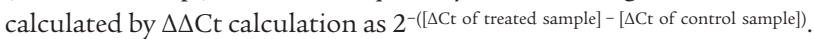

ELISAs. Secretion of TNF- $\alpha$ and MCP-1 proteins from macrophages and MLECs was determined by using the Quantikine ELISA kits from $\mathrm{R} \& \mathrm{D}$ Systems.

Statistics. Unless otherwise mentioned, data are expressed as mean \pm SEM. Unpaired 2-tailed Student's $t$ test was performed for comparisons between 2 groups. For multiple comparison, 2-way ANOVA was followed by Bonferroni's test to determine statistical significance by using GraphPad Prism software. $P<0.05$ was considered to be statistically significant.

\section{Acknowledgments}

We thank M. Sasaki for helpful comments on histological analysis. We thank M.Okabe for the generous gift of GFP mice and S. Kaneko for the use of a cell sorter. We also thank C. Hirose and Y. Mishima for secretarial and technical assistance. This work was supported by grants from the Ministry of Education, Science, Sports, and Culture of Japan, and the Japan Society for the Promotion of Science, and a research grant from AstraZeneca.

Received for publication January 13, 2010, and accepted in revised form September 1, 2010.

Address correspondence to: Yoh Takuwa or Yasuo Okamoto, Department of Physiology, Graduate School of Medicine, Kanazawa University, 13-1 Takara-machi, Kanazawa, Ishikawa 920-8640, Japan. Phone: 81.76.265.2165; Fax: 81.76.234.4223; E-mail: ytakuwa@med.kanazawa-u.ac.jp (Y. Takuwa); linakura@ med.kanazawa-u.ac.jp (Y. Okamoto).
1. Rader DJ, Daugherty A. Translating molecular discoveries into new therapies for atherosclerosis. Nature. 2008;451(7181):904-913.

2. Libby P. Inflammation in atherosclerosis. Nature.
2002;420(6917):868-874

3. Ishii I, Fukushima N, Ye X, Chun J. Lysophospholipid receptors: signaling and biology. Annu Rev Biochem. 2004;73:321-354.
4. Nofer JR, Assmann G. Atheroprotective effects of high-density lipoprotein-associated lysosphingolipids. Trends Cardiovasc Med. 2005;15(7):265-271.

5. Schwab SR, Pereira JP, Matloubian M, Xu Y, Huang Y, 
Cyster JG. Lymphocyte sequestration through S1P lyase inhibition and disruption of S1P gradients. Science. 2005;309(5741):1735-1739.

6. Venkataraman K, et al. Vascular endothelium as a contributor of plasma sphingosine 1-phosphate. Circ Res. 2008;102(6):669-676.

7. Spiegel S, Milstien S. Sphingosine-1-phosphate: an enigmatic signalling lipid. Nat Rev Mol Cell Biol. 2003;4(5):397-407.

8. Pappu R, et al. Promotion of lymphocyte egress into blood and lymph by distinct sources of sphingosine1-phosphate. Science. 2007;316(5822):295-298.

9. Li Z, et al. Regulation of PTEN by Rho small GTPases. Nat Cell Biol. 2005;7(4):399-404.

10. Ryu Y, et al. Sphingosine-1-phosphate, a plateletderived lysophospholipid mediator, negatively regulates cellular Rac activity and cell migration in vascular smooth muscle cells. Circ Res. 2002;90(3):325-332.

11. Inoki I, et al. Negative regulation of endothelial morphogenesis and angiogenesis by S1P2 receptor. Biochem Biophys Res Commun. 2006;346(1):293-300.

12. Du W, et al. $S 1 P_{2}$, the $G$ protein-coupled receptor for sphingosine-1-phosphate, negatively regulates tumor angiogenesis and tumor growth in vivo in mice. Cancer Res. 2010;70(2):772-781.

13. Hughes JE, Srinivasan S, Lynch KR, Proia RL, Ferdek $\mathrm{P}$, Hedrick CC. Sphingosine-1-phosphate induces an antiinflammatory phenotype in macrophages. Circ Res. 2008;102(8):950-958.

14. Whetzel AM, et al. Sphingosine-1 phosphate prevents monocyte/endothelial interactions in type 1 diabetic NOD mice through activation of the S1P1 receptor. Circ Res. 2006;99(7):731-739.

15. Nofer JR, et al. FTY720, a synthetic sphingosine 1 phosphate analogue, inhibits development of atherosclerosis in low-density lipoprotein receptordeficient mice. Circulation. 2007;115(4):501-508.

16. Keul P, et al. The sphingosine-1-phosphate analogue FTY720 reduces atherosclerosis in apolipoprotein E-deficient mice. Arterioscler Thromb Vasc Biol. 2007;27(3):607-613.

17. Mallat Z, et al. Rho-associated protein kinase contributes to early atherosclerotic lesion formation in mice. Circ Res. 2003;93(9):884-888.

18. Wang HW, et al. Deficiency of ROCK1 in bone marrow-derived cells protects against atherosclerosis in LDLR $^{-/-}$mice. FASEB J. 2008;22(10):3561-3570.

19. MacLennan AJ, et al. An essential role for the H218/ AGR16/Edg-5/LP(B2) sphingosine 1-phosphate receptor in neuronal excitability. Eur J Neurosci. 2001;14(2):203-209.

20. Kuhlencordt PJ, et al. Accelerated atherosclerosis, aortic aneurysm formation, and ischemic heart disease in apolipoprotein E/endothelial nitric oxide synthase double-knockout mice. Circulation. 2001;104(4):448-454.

21. Ricci R, et al. Requirement of JNK2 for scavenger receptor A-mediated foam cell formation in atherogenesis. Science. 2004;306(5701):1558-1561.

22. Kunjathoor VV, et al. Scavenger receptors class A-I/II and $\mathrm{CD} 36$ are the principal receptors responsible for the uptake of modified low density lipoprotein leading to lipid loading in macrophages. J Biol Chem. 2002;277(51):49982-49988
23. Yvan-Charvet L, et al. Combined deficiency of ABCA1 and ABCG1 promotes foam cell accumulation and accelerates atherosclerosis in mice. J Clin Invest. 2007;117(12):3900-3908.

24. Tall AR, Yvan-Charvet L, Terasaka N, Pagler T, Wang N. HDL, ABC transporters, and cholesterol efflux: implications for the treatment of atherosclerosis. Cell Metab. 2008;7(5):365-375.

25 . Bradley MN, et al. Ligand activation of LXR beta reverses atherosclerosis and cellular cholesterol overload in mice lacking LXR alpha and apoE. J Clin Invest. 2007;117(8):2337-2346.

26. Zelcer N, Tontonoz P. Liver X receptors as integrators of metabolic and inflammatory signaling. J Clin Invest. 2006;116(3):607-614.

27. Tabas I. Consequences of cellular cholesterol accumulation: basic concepts and physiological implications. J Clin Invest. 2002;110(7):905-911.

28. Sugimoto N, Takuwa N, Okamoto H, Sakurada S, Takuwa Y. Inhibitory and stimulatory regulation of Rac and cell motility by the G12/13-Rho and Gi pathways integrated downstream of a single $G$ protein-coupled sphingosine-1-phosphate receptor isoform. Mol Cell Biol. 2003;23(5):1534-1545.

29. Anwar KN, Fazal F, Malik AB, Rahman A. RhoA/ Rho-associated kinase pathway selectively regulates thrombin-induced intercellular adhesion molecule- 1 expression in endothelial cells via activation of I kappa B kinase beta and phosphorylation of RelA/p65. J Immunol. 2004;173(11):6965-6972.

30. Zhou Q, Liao JK. Rho kinase: an important mediator of atherosclerosis and vascular disease. Curr Pharm Des. 2009;15(27):3108-3115.

31. Ozawa K, Hirata K, Yamamoto K, inventors; Japan Tobacco Inc, assignee. Pyrazolopyridine derivatives and medicinal use thereof. World Intellectual Property Organization patent WO/2003/051876 A1. June 26, 2003

32. Argmann CA, et al. Regulation of macrophage cholesterol efflux through hydroxymethylglutaryl-CoA reductase inhibition: a role for RhoA in ABCA1-mediated cholesterol efflux. J Biol Chem. 2005;280(23):22212-22221

33. Park YM, Febbraio M, Silverstein RL. CD36 modulates migration of mouse and human macrophages in response to oxidized LDL and may contribute to macrophage trapping in the arterial intima. J Clin Invest. 2009;119(1):136-145.

34. Swirski FK, et al. Ly-6Chi monocytes dominate hypercholesterolemia-associated monocytosis and give rise to macrophages in atheromata. J Clin Invest. 2007;117(1):195-205

35. Ross R. Atherosclerosis--an inflammatory disease. NEngl J Med. 1999;340(2):115-126.

36. Laufs U, La Fata V, Plutzky J, Liao JK. Upregulation of endothelial nitric oxide synthase by HMG CoA reductase inhibitors. Circulation. 1998;97(12):1129-1135.

37. Lin CI, Chen CN, Lin PW, Lee H. Sphingosine 1-phosphate regulates inflammation-related genes in human endothelial cells through S1P1 and S1P3. Biochem Biophys Res Commun. 2007; 355(4):895-901.

38. Wolfrum S, et al. Inhibition of Rho-kinase leads to rapid activation of phosphatidylinositol 3-kinase/ protein kinase Akt and cardiovascular protection.
Arterioscler Thromb Vasc Biol. 2004;24(10):1842-1847.

39. Benitah SA, Valeron PF, Lacal JC. ROCK and nuclear factor-kappaB-dependent activation of cyclooxygenase- 2 by Rho GTPases: effects on tumor growth and therapeutic consequences. Mol Biol Cell. 2003;14(7):3041-3054.

40. Lepley D, Paik JH, Hla T, Ferrer F. The G proteincoupled receptor S1P2 regulates Rho/Rho kinase pathway to inhibit tumor cell migration. Cancer Res. 2005;65(9):3788-3795.

41. Bot I, et al. Perivascular mast cells promote atherogenesis and induce plaque destabilization in apolipoprotein E-deficient mice. Circulation. 2007;115(19):2516-2525

42. Nofer JR, et al. HDL induces NO-dependent vasorelaxation via the lysophospholipid receptor S1P3. J Clin Invest. 2004;113(4):569-581.

43. Fernandez-Hernando C, et al. Loss of Akt1 leads to severe atherosclerosis and occlusive coronary artery disease. Cell Metab. 2007;6(6):446-457.

44. Gu L, et al. Absence of monocyte chemoattractant protein-1 reduces atherosclerosis in low density lipoprotein receptor-deficient mice. Mol Cell. 1998;2(2):275-281.

45. Boring L, Gosling J, Cleary M, Charo IF. Decreased lesion formation in CCR2 $2^{-/}$mice reveals a role for chemokines in the initiation of atherosclerosis. Nature. 1998;394(6696):894-897.

46. Zhu SN, Chen M, Jongstra-Bilen J, Cybulsky MI. GM-CSF regulates intimal cell proliferation in nascent atherosclerotic lesions. J Exp Med. 2009;206(10):2141-2149

47. Tabas I. Consequences and therapeutic implications of macrophage apoptosis in atherosclerosis: the importance of lesion stage and phagocytic efficiency. Arterioscler Thromb Vasc Biol. 2005; 25(11):2255-2264.

48. Han S, et al. Macrophage insulin receptor deficiency increases ER stress-induced apoptosis and necrotic core formation in advanced atherosclerotic lesions. Cell Metab. 2006;3(4):257-266.

49. Libby P, Aikawa M. Stabilization of atherosclerotic plaques: new mechanisms and clinical targets. Nat Med. 2002;8(11):1257-1262.

50. Aiello RJ, Bourassa PA, Lindsey S, Weng W, Freeman A, Showell HJ. Leukotriene B4 receptor antagonism reduces monocytic foam cells in mice. Arterioscler Thromb Vasc Biol. 2002;22(3):443-449.

51 . Veillard NR, et al. Antagonism of RANTES receptors reduces atherosclerotic plaque formation in mice. Circ Res. 2004;94(2):253-261.

52. Tsukamoto K, et al. Synergically increased expression of CD36, CLA-1 and CD68, but not of SR-A and LOX-1, with the progression to foam cells from macrophages. J Atheroscler Thromb. 2002;9(1):57-64.

53. Ohkawa R, et al. Plasma sphingosine-1-phosphate measurement in healthy subjects: close correlation with red blood cell parameters. Ann Clin Biochem. 2008;45(pt 4):356-363.

54. Hahn CG, et al. In vivo and in vitro neurogenesis in human olfactory epithelium. J Comp Neurol. 2005; 483(2):154-163.

55. Gareus R, et al. Endothelial cell-specific NF-kap$\mathrm{paB}$ inhibition protects mice from atherosclerosis. Cell Metab. 2008;8(5):372-383. 